\title{
Proof of Chiral Symmetry Breaking in Strongly Coupled Lattice Gauge Theory
}

\author{
Manfred Salmhofer * and Erhard Seiler \\ Max-Planck-Institut für Physik und Astrophysik, Werner-Heisenberg Institut, \\ Föhringer Ring 6, W-8000 München 40, Federal Republic of Germany
}

Received January 8, 1991

\begin{abstract}
We study chiral symmetry in the strong coupling limit of lattice gauge theory with staggered fermions and show rigorously that chiral symmetry is broken spontaneously in massless QED and the gauge-invariant Nambu-Jona-Lasinio model if the dimension of spacetime is at least four. The results for the chiral condensate as a function of the mass imply that the mean-field approximation is an upper bound for this observable which becomes exact as the dimension goes to infinity. For the model with gauge group $U(N), N=2,3,4$, we prove that chiral long-range order exists at zero mass in four or more dimensions.
\end{abstract}

\section{Introduction}

The idea that the pion is an approximate Nambu-Goldstone particle induced by the (approximately) spontaneous breaking of the axial $S U(2)$ invariance, and that the same mechanism gives most of its mass to the nucleon, is by now thirty years old $[1,2]$. It has survived the tremendous changes that have occurred since then in the description of strong interactions and is nowadays generally believed to be realized in QCD. Nevertheless to this day a more rigorous mathematical understanding of this phenomenon, going beyond the mean-field analysis in [2] is lacking.

Heuristic arguments that were advanced much more recently suggested that spontaneous breaking of chiral symmetry should be in some way related to confinement in QCD with massless quarks [3]; these arguments still hinge on the relation of chiral symmetry breaking and condensation phenomena that was for purely fermionic theories first analyzed in the above-mentioned pioneering papers by Nambu and Jona-Lasinio $[1,2]$. In their treatment the

\footnotetext{
* Address after August 1991: Mathematics Department, University of British Columbia, Vancouver, Canada V6T1Y4
} 
nucleons appear as "dressed" particles and their mass is proportional to the value of the chiral condensate of a fundamental theory of massless fermions alone, which exhibits chiral symmetry breaking. Mesons arise as bound states from a condensation of the fundamental fermions. The Nambu-Jona-Lasinio (NJL) model is not renormalizable, which makes a treatment surmounting the mean-field-approximation of Nambu and Jona-Lasinio difficult, but its two-dimensional analogue, first introduced by Thirring [4], is [5], and chiral symmetry breaking was shown to take place in this model [6] in the $N=\infty$ limit. However, there remained the question how this result could be reconciled with the Mermin-Wagner [7, 8] or Coleman theorem [9] which states that continuous symmetries cannot be broken spontaneously in two-dimensional systems of statistical mechanics or relativistic quantum field theory.

Recently, the question of spontaneous chiral symmetry breaking has played a role in the triviality discussion of QED [10-16]. Miransky [10] solved a truncated version of the Schwinger-Dyson equations which is obtained by omission of vertex corrections and fermion loops. In this approximation there is a critical value $e_{c}$ of the bare charge $e_{0}$ such that chiral symmetry is broken for $e_{0}>e_{c}$, and $e_{c}$ is a point where the ultraviolet cutoff can be removed. Miransky also gave a scaling prediction for the continuum theory to be constructed at $e_{c}$. Apart from other objections, his truncation (as well as the improved one of Rakow [17]) of the Schwinger-Dyson equations is inconsistent with the Ward identity and the results so obtained seem seriously defective.

Since chiral symmetry breaking was claimed to happen at large values of $e_{0}$, it is natural to consider the question in the framework of lattice gauge theory, where the problems with the Ward identity are absent because the regularization of the interaction between gauge fields and fermions is gauge-invariant in this case. Kogut, Dagotto and Kocic [11], and later on Schierholz and coworkers $[12,14]$, did numerical studies to analyze the phase structure of QED with staggered fermions. Staggered fermions where chosen because they still have a continuous chiral symmetry; moreover, they are technically simpler than the physically more desirable Wilson fermions. The numerical results agree with Miransky's as far as the existence of $e_{c}$ and a chiral-symmetry-breaking phase at strong coupling is concerned, and the transition at $e_{c}$ seems to be of second order at least for the noncompact gauge field integral. The scaling prediction of Miransky, seemingly consistent with the data at first, is ruled out by now, but there is still an ongoing controversy as to whether the critical exponents take their mean-field values or not. The discussion has also renewed the interest in the gauge-invariant version of the NJL model because the four-fermion-interactions were claimed to become renormalizable due to large anomalous dimensions, and also more technically because they play a role in the renormalization group flow of lattice QED.

To the best of our knowledge, not many rigorous results about lattice models with fermions have been obtained outside the cluster expansion region, and in particular there seems to be no result about chiral symmetry breaking in the literature. For QED, Lüscher has proven that the charge is always screened, $0 \leqq Z_{3} \leqq 1$ for all $e_{0}$, and assuming chiral symmetry breaking to hold and also using the numerical prediction for $e_{c}$ he gives arguments for triviality [15].

We present here a proof of chiral symmetry breaking in the strong coupling limit of lattice gauge theories with staggered fermions, which applies to QED, 
$U(N)$-models for $N \leqq 4$ in four or more dimensions, and also to the NambuJona-Lasinio type models obtained by including a four-fermion term in the action. The rigorous results about strongly coupled QED are: for all $m>0$ there is no phase transition and exponential clustering holds for truncated correlations. If the dimension is at least four, there is a phase transition at $m=0$, namely the limit of the chiral condensate as the mass vanishes is nonzero. The mean field approximation for this observable is an upper bound in all dimensions which becomes exact as the dimension tends to infinity. Whenever the chiral condensate has a nonzero limit there is also chiral long-range order in the system, and we can show that chiral long-range order exists also in the more complicated $U(N)$-models. Our results are still very far from giving a rigorous underpinning to the old ideas of chiral symmetry breaking in strong interactions, but to our knowledge they are the first rigorous results on chiral symmetry breaking in existence.

The techniques used to derive these results are the infrared bounds of Fröhlich, Simon, and Spencer [18, 19] and some Schwinger-Dyson-type equations. There were technical problems to be solved due to the fact that even though the fermionic system can be rewritten as a spin system in the limit we consider, this spin model is inherently complex and thus not accessible to probabilistic methods. The fact that the action is complex also implies that the Fourier transform of the two-point function $\hat{T}$ can become negative (and chiral symmetry in finite volume forces it to do so); therefore it is not a priori clear that it is a measure, which is a necessary ingredient for the proof of chiral symmetry breaking. It is again the infrared bound which provides not only the upper bound, but also a lower one by which we can show that $\hat{T}$ is indeed a measure in the thermodynamic limit.

The infrared bound method works also in three dimensions, and it is fair to ask why our statement starts with dimension four. This is not a principal restriction but rather a technical one; the bounds for the fluctuations are a little too weak in three dimensions in the case of QED. For a more general complex spin system, called NJL system in the sequel, which for $N=1$ is identical to QED (with or without a four-fermion-interaction), chiral symmetry breaking can also be shown to occur in three dimensions, if $N \geqq 2$. For $N \geqq 2$ NJL systems have a chirally invariant current interaction. These systems can alternatively be represented as monomer-dimer models which makes it possible to use the strong results obtained in $[20,21]$. While in one dimension the system is equivalent to a free fermion system, and exhibits chiral symmetry breaking, we are not able to calculate the limit of the chiral condensate in two dimensions. However, an old result of Fisher and Stephenson [22] shows that the two-point function clusters at zero mass in two dimensions. While there is no extension of the Mermin-Wagner theorem to these systems (in particular because chiral symmetry is broken in one dimension), it is likely that the symmetry remains unbroken in that case. This also agrees with the result one gets in the continuum Schwinger model with two flavours, and thus with the usual interpretation of the remaining degeneracy of staggered fermions as flavours.

The result for four-dimensional QED means that there is a phase of the model with staggered fermions in which chiral symmetry is broken. To establish the existence of the phase transition when the gauge coupling is varied, one still has to show that the symmetry is unbroken at weak coupling, which is of course very plausible, but as yet unproven. 
We define the models and order parameters in Sect. 2, and recall how the strongly coupled system can be mapped to a complex spin system. Systems of this kind are studied in Sect. 3, both directly and in their monomer-dimer representation. Section 4 comprises the results about chiral symmetry breaking, and the appendix contains some technical details used to bound the fluctuations.

\section{Models and Order Parameters}

We consider compact and noncompact QED on the lattice as well as compact $U(N)$-gauge theory $(N \geqq 2)$ coupled to staggered fermions and gauged four-fermion models which can be obtained by addition of a four-fermion interaction term. Let $\Lambda \subset \mathbb{Z}^{v},|\Lambda|<\infty$ and for $x \in \Lambda, \psi_{a}(x)$ and $\psi_{a}(x)$ be Grassmann variables carrying a vector index $a \in\{1, \ldots, N\}$ of the fundamental representation of $U(N)$ acting on the $\psi$ and $\bar{\psi}$. The gauge fields are represented by the parallel transporters $U_{\mu}(x) \in U(N)$ associated to each link. The lattice will be taken as a block

$$
\Lambda=\left\{x=\left(x_{1}, \ldots, x_{v}\right) \in \mathbb{Z}^{v} \mid \forall i \in\{1, \ldots, v\}: 0 \leqq x_{i}<L_{i}\right\}
$$

with sidelengths $L_{i} \in \mathbb{N}$ and free or, more often, toroidal boundary conditions are chosen; toroidal means periodic for the gauge field and antiperiodic for the fermion fields. The action is

$$
S=S_{F}+\beta S_{g}+g_{4} S_{4}
$$

where $g_{4} \geqq 0$,

$$
\begin{aligned}
S_{F}= & \sum_{x \in A}\left(\frac{1}{2} \sum_{\mu} \Gamma_{\mu}(x)\left(\bar{\psi}(x) U_{\mu}(x) \psi\left(x+e_{\mu}\right)-\bar{\psi}\left(x+e_{\mu}\right) U_{\mu}(x)^{-1} \psi(x)\right)\right. \\
& -m \bar{\psi}(x) \psi(x))
\end{aligned}
$$

and the $\gamma$ matrices are replaced by the sign factors

$$
\Gamma_{\mu}(x)=(-1)^{\sum_{\mathrm{e}=1}^{\mu-1} x_{e}}
$$

For technical and interpretational reasons, we will always assume that $L_{\mu} \in 2 \mathbb{N}$ for all $\mu \in\{1, \ldots, v\}$. If the boundary conditions are toroidal, one would get into consistency troubles with the various sign factors $\Gamma_{\mu}$ and $\varepsilon(x)$ if one refused to bend to this restriction.

$S_{4}$ is the gauge invariant four-fermion interaction

where

$$
S_{4}=-\sum_{x \in \Lambda} \sum_{\mu=1}^{v} \bar{\psi} \psi(x) \bar{\psi} \psi\left(x+e_{\mu}\right)
$$

$$
\bar{\psi} \psi(x)=\sum_{a=1}^{N} \bar{\psi}_{a}(x) \psi_{a}(x) .
$$

Chiral invariance holds in the form that at mass $m=0$, for all $\beta$ and $g_{4}$, the action is invariant under the transformation

$$
\begin{aligned}
& \psi(x) \mapsto e^{i \alpha \varepsilon(x)} \psi(x) \\
& \bar{\psi}(x) \mapsto e^{i \alpha \varepsilon(x)} \bar{\psi}(x)
\end{aligned}
$$


with a continuous parameter $\alpha$ and the sign factor

$$
\varepsilon(x)=(-1)^{\sum_{\mu=1}^{v} x_{\mu}}
$$

which plays the role of $\gamma_{5}$ for staggered fermions. This chiral invariance would of course have been spoiled for $g_{4}>0$ if a strictly local four-fermion term of the type $\bar{\psi} \psi(x)^{2}$ had been introduced for $N>1$ (for $N=1$ it would be identically zero because of nilpotency).

While technically in several respects convenient, staggered fermions still do not seem to be satisfactorily understood as far as their interpretation is concerned. That only one spinor component sits at every lattice site is not the real obstacle because Dirac and spacetime indices are somewhat mixed in this formalism; to establish the connection to the continuum one has to introduce blocks on the lattice and construct spinors from the fermions by taking their components to be the fields at different points in the same block [23]. It remains an open question, however, whether the remaining degeneracy can then really be interpreted as occurrence of flavours. Sharatchandra, Thun and Weisz [24] have shown that it is also possible to define a current which has the anomaly familiar from continuum perturbation theory. This current is, of course, not directly related to the symmetry called chiral, (2.7).

The gauge part $S_{g}$ of the action is multiplied by $\beta=\frac{1}{e_{0}^{2}}$; here it is taken in Wilson's original form which reproduces the continuum action in the limit of vanishing lattice spacing [25].

The partition function is defined as

$$
Z_{\Lambda}=\int \mathscr{D}_{\Lambda} \psi \bar{\psi} \mathscr{D}_{\Lambda} U e^{-S}
$$

and expectation values of functions $f(U, \psi, \bar{\psi})$ by

$$
\langle f\rangle_{S, \Lambda}=\frac{1}{Z_{\Lambda}} \int \mathscr{D}_{\Lambda} \psi \bar{\psi} \mathscr{D}_{\Lambda} U e^{-S} f(U, \psi, \bar{\psi}) .
$$

In these expressions, the fermionic integral [26] is

and

$$
\int \mathscr{D}_{\Lambda} \psi \bar{\psi}=\int \prod_{x \in \Lambda} \prod_{a=1}^{N} d \psi_{a}(x) d \bar{\psi}_{a}(x),
$$

$$
\mathscr{D}_{\Lambda} U=\prod_{x \in \Lambda} \prod_{\mu=1}^{v} d U_{\mu}(x),
$$

where $d U$ denotes the Haar measure on $U(N)$.

For $N=1, \mathrm{QED}$, using the parametrization $U_{\mu}(x)=e^{i A_{\mu}(x)}$ with $A_{\mu}(x) \in \mathbb{R}$, the group integral amounts to integrating $A_{\mu}(x)$ from $-\pi$ to $\pi$. One can also define a "noncompact" regularization of the theory where $A$ is integrated over the whole real axis and the action is the discretization of the continuum fieldstrength term together with a gauge fixing term. Bałaban, Brydges, Imbrie and Jaffe [27] have shown that the expectation values of gauge invariant functions of the fields which are periodic in $A$ in the noncompact model coincide with those of compact QED with a special action containing vortex fields. This statement is also true if the boundary conditions are chosen toroidal [28], and it implies that compact and noncompact QED are identical in the strong 
coupling limit $\beta=0$, so our results also apply to the noncompact theory. Suitable gauge fixing terms include the usual $\left(d^{*} A\right)^{2}$-term with compact integration over the zero modes as a special case (the zero modes cannot simply be fixed, of course), and it is also identical to what one gets from a limit of a gauge fixing term which regularizes the zero-mode divergences by a mass, as this mass is sent to zero [28]. If $|\Lambda|<\infty, \lim _{m \rightarrow 0}\langle\bar{\psi} \psi(x)\rangle_{\Lambda}=0$ because of continuity.

Definition 2.1. The chiral order parameter is

$$
X=\liminf _{m \rightarrow 0}\langle\bar{\psi} \psi(x)\rangle=\liminf _{m \rightarrow 0} \lim _{\Lambda \rightarrow \infty}\langle\bar{\psi} \psi(x)\rangle_{\Lambda} .
$$

There is long-range order (LRO) in the system if there is a truncated correlation function which does not cluster at zero mass, e.g. if

$$
\limsup _{|x| \rightarrow \infty}\langle\bar{\psi} \psi(0) \bar{\psi} \psi(x)\rangle_{m=0} \neq 0
$$

(because $\langle\bar{\psi} \psi(x)\rangle_{m=0}=0$ ).

Remark 2.2. For free $(\beta=\infty)$ staggered or naive fermions, chiral symmetry is broken only in one dimension,

$$
X=\left\{\begin{array}{ll}
N & \text { for } v=1 \\
0 & \text { for } v \geqq 2
\end{array} .\right.
$$

This means simply that there are two states of zero energy for $v=1$.

In classical statistical mechanics, violation of clustering implies that the infinite volume state constructed at $m=0$ is a mixture of coexisting phases, so that there must be a phase transition at this point [29]. If the chirally invariant two-point function is continuous at $m=0$ and clustering holds at $m>0, X \neq 0$ and (2.14) are equivalent.

\section{Gauge Field Integration at Strong Coupling}

In the strong coupling limit $\beta \rightarrow 0\left(e_{0} \rightarrow \infty\right)$ the gauge field is "completely random" and the integral over the gauge fields can be done, leaving behind an effective action for the matter fields. This effective action can in principle be controlled by cluster expansion methods for $0 \leqq \beta \leqq \beta_{0}$ but is nonlocal if $\beta>0$. At $\beta=0$, however, the gauge integration can be done separately on each link and it generates an interaction between fields on adjacent sites only.

An explicit expression for this nearest-neighbour-interaction was calculated for staggered fermions by Rossi and Wolff [30]. Staggered fermions seem to be the only case where this is possible; for fermions with more than one spinor component per lattice site no simple expressions are available, but many papers on the formal $1 / N$-expansion for the effective action of $U(N)$-theory and the corresponding expansion around mean field theory have been published [31-34]. The conditions under which we have a proof that the expansion for the effective action is mathematically controllable are naturally fulfilled for gauge theories with bosonic matter [28], but we cannot bound the remainders for fermions. For staggered fermions the exponential of the effective action is [30]

$$
\widetilde{B}(t)=\sum_{k=0}^{N} \tilde{b_{k}} t^{k}
$$


where

$$
\tilde{b_{k}}=\frac{(N-k) !}{N ! k !}
$$

The effective interaction $\tilde{W}$ is defined in terms of $\widetilde{B}$ as

$$
\tilde{W}(t)=\log \tilde{B}(t),
$$

this definition makes sense as a formal power series in $t$ because $\widetilde{B}(0)=1$. Moreover the element of the Grassmann algebra which is the argument of $W$ is nilpotent, therefore the series for the logarithm terminates after a finite number of terms. The partition function can thus be rewritten in the strong coupling limit $\beta=0$ as that of a purely fermionic system,

$$
Z_{\Lambda}=\int \mathscr{D}_{\Lambda} \psi \bar{\psi} \exp \left(\sum_{x} m \bar{\psi} \psi(x)+\sum_{x, \mu} \tilde{W}\left(\frac{1}{4} \bar{\psi} \psi(x) \bar{\psi} \psi\left(x+e_{\mu}\right)\right)\right)
$$

and so can observables. The action depends only on the bilinears $\bar{\psi} \psi(x)$ which can be interpreted as mesonic fields; this allows to do a bosonization which results in a complex spin system, using the formula [30]

$$
\int d \psi d \bar{\psi} f(\bar{\psi} \psi)=N ! \oint \frac{d \tilde{\sigma}}{2 \pi i \tilde{\sigma}} \tilde{\sigma}^{-N} f(\tilde{\sigma})
$$

for the Grassmann integral at each lattice point. It is convenient to rescale and call $\sigma_{x}=\tilde{\sigma} / 2 N$, motivated by the fact that $\tilde{\sigma}$ replaces $\sum_{a=1}^{N} \bar{\psi}_{a} \psi_{a}$. Dropping global constants,

$$
\langle F\rangle_{\Lambda}=\frac{1}{Z_{\Lambda}} \oint\left(\prod_{x \in \Lambda} \frac{d \sigma_{x}}{2 \pi i \sigma_{x}} \sigma_{x}^{-N} e^{2 N m \sigma_{x}}\right) e^{N \sum_{x, \mu} W\left(\sigma_{x} \sigma_{x+e_{\mu}}\right)},
$$

where

$$
W(t)=\frac{1}{N} \tilde{W}\left(N^{2} t\right)=\sum_{k=0}^{N} w_{k} t^{k}
$$

is the interaction and

$$
\begin{aligned}
B(t) & =e^{N W(t)}=e^{4 N g_{4} t} \sum_{k=0}^{N} b_{k} t^{k}, \\
b_{k} & =\frac{(N-k) !}{N ! k !} N^{2 k},
\end{aligned}
$$

its exponential. In particular, $w_{1}=1+4 N g_{4}$. The expectation value of $\bar{\psi} \psi$ is given by

$$
\langle\bar{\psi} \psi(x)\rangle_{\Lambda}=2 N\left\langle\sigma_{x}\right\rangle_{\Lambda},
$$

therefore the chiral order parameter is

$$
X=2 N \liminf _{m \rightarrow 0}\left\langle\sigma_{x}\right\rangle .
$$

\section{Complex Spin Systems and Monomer-Dimer Models}

A general framework for the rewritings of fermionic systems at strong coupling is given by 
Definition 3.1. Let $N \in \mathbb{N}$ and $G$ be a graph with finite vertex set $V(G)$ and $a$ set $B(G)$ of bonds $i=(x, y)$ connecting vertices $x$ and $y$. To each vertex $x \in V(G)$ assign a complex variable (spin) $\sigma_{x}$ and a function $F_{x}: \mathbb{C} \rightarrow \mathbb{C}$ which is analytic at zero. To each bond $i \in B(G)$, assign a function $B_{i}: \mathbb{C} \rightarrow \mathbb{C}$ which is also analytic at zero and satisfies $B_{i}(0)=1$. The complex spin system on $G$ is defined by the partition function

$$
Z_{G}=\oint\left(\prod_{x \in V(G)} \frac{d \sigma_{x}}{2 \pi i \sigma_{x}} \sigma_{x}^{-N} F_{x}\left(\sigma_{x}\right)\right) \prod_{i=(x, y) \in B(G)} B_{i}\left(\sigma_{x} \sigma_{y}\right)
$$

and for $Z_{G} \neq 0$, expectation values of functions $\phi$ which are analytic at zero in all $\sigma_{x}$ are defined by

$$
\begin{gathered}
\langle\phi\rangle_{G}=\frac{1}{Z_{G}} \oint\left(\prod_{x \in V(G)} \frac{d \sigma_{x}}{2 \pi i \sigma_{x}} \sigma_{x}^{-N} F_{x}\left(\sigma_{x}\right)\right) \phi(\sigma) \prod_{i=(x, y) \in B(G)} B_{i}\left(\sigma_{x} \sigma_{y}\right) . \\
W_{x y}(t)=\frac{1}{N} \log B_{x y}(t)
\end{gathered}
$$

is called the interaction of the system.

Remark 3.2. In Definition 3.1 the path of integration was not specified; it is of course assumed to be chosen at each $x$ such that it fits into the common domain of analyticity of $F_{x}$ and all $B_{i}$ with $x \in i$. However, it is clear that $F_{x}$ and $B_{i}$ can then be replaced by the polynomials one gets when truncating their Taylor expansion after $N$ terms without changing the result, and since polynomials are entire functions the path of integration can then be chosen arbitrarily (around zero). This truncation will always be assumed to have been done and the path of integration will always be taken to be the unit circle. The fact that only polynomials matter corresponds to the nilpotency of the original Grassmann variables. This choice of the path means for staggered fermions that the chiral transformations are represented in the associated complex spin system by

$$
\sigma_{x} \rightarrow e^{2 i \alpha \varepsilon(x)} \sigma_{x} .
$$

\section{Map to a Monomer-Dimer Model}

Complex spin systems can be mapped to monomer-dimer models simply by expansion of all the functions and calculation of the contour integral at each lattice point. The residue is only nonzero for a fixed combination of powers occurring in the expansion; this gives rise to a hard-core constraint for the associated monomer-dimer model.

For $N=1$ and $F_{x}\left(\sigma_{x}\right)=e^{m_{x} \sigma_{x}}$ the partition function turns out to be the classical monomer-dimer system $[35,20,21]$ which is one of the oldest models in statistical mechanics and has a simple geometric interpretation: a monomer is an object which can occupy a vertex, dimers can sit on bonds. The activity of a monomer at $x$ is $m_{x}$ and that of a dimer on $i=(x, y)$ is $w_{x y}$, where

$$
B_{x y}(t)=e^{W_{x y}(t)}=1+w_{x y} t+O\left(t^{2}\right)
$$


(the higher orders in $t$ do not have any influence, see Remark 3.2). An allowed dimer configuration is an arrangement of dimers on the bonds of $G$ such that no two dimers can have a vertex in common: they have a hard-core-interaction. For an allowed dimer configuration $C$, denote

$$
D_{C}=\prod w_{i},
$$

where the product is over all edge weights of occupied bonds $i \in B(G)$. Thus the expression for the partition function can be restated as a sum over all allowed dimer configurations on $G$,

$$
Z_{G}=\sum_{C} D_{C} \prod m_{x}
$$

with monomers filled in at all those vertices that are not touched by a dimer. The restriction to allowed dimer configurations as well as the filling condition come from the contour integral.

If $F_{x}\left(\sigma_{x}\right)=e^{N m_{x} \sigma_{x}}$ but the interaction $W$ is derived from the $U(N)$-model with $N>1$, the system is more complicated; it may be called a generalized monomer-dimer model and its rules are discussed in [30]. The difference between generalized and ordinary monomer-dimer models is, however, rather due to the type of interaction $W$ than to the value of $N$.

Definition 3.3. Let $N \in \mathbb{N}, F_{x}(z)=e^{N m_{x} z}, B_{x y}(z)=e^{N W_{x y}(z)}$.

(1) If $W_{x y}(z)=w_{1} z$ for $(x, y) \in B(G), w_{1}>0$, the complex spin system is called a NJL system.

(2) The system given by

$$
\begin{aligned}
B(t) & =e^{N W(t)}=e^{4 N g_{4} t} \sum_{k=1}^{N} b_{k} t^{k}, \\
b_{k} & =\frac{(N-k) !}{N ! k !} N^{2 k}
\end{aligned}
$$

on all bonds is called a $U(N)$-system.

(3) The NJL- $\left(U(N)\right.$-) system on $\Lambda \subset \mathbb{Z}^{v}$ is the system on the graph with vertex set $\Lambda$ and set of bonds $B=\{(x, y):|x-y|=1\}$, with $m_{x}=m$ on all vertices and $W_{x y}(t)=w_{1} t$ (respectively $W_{x y}(t)=W(t)$ with $\exp W(t)=B(t), B(t)$ defined by (3.8)) on all bonds $(x, y) \in B$.

If one truncates the interaction $W(t)$ after the first term in the $U(N)$-system, one gets an NJL-system, for this reason the NJL systems were called "simplified" in [36].

Remark 3.4. For all $g_{4} \geqq 0$ :

(1) Strongly coupled QED is an NJL system.

(2) Strongly coupled $U(N)$-lattice gauge theory is a $U(N)$-system.

(3) The NJL system on $G$ can be mapped to an ordinary monomer-dimersystem on a graph with vertex set $V(G) \times\{1, \ldots, N\}$.

(4) By trivial rescaling, without loss of generality, $w_{1}=1$. 
Proof of (3). The partition function is

$$
Z_{G}=\oint\left(\prod_{x \in V(G)} \frac{d \sigma_{x}}{2 \pi i \sigma_{x}} \sigma_{x}^{-N} e^{m_{x} \sigma_{x}}\right) \prod_{i=(x, y) \in B(G)} e^{w_{x y} \sigma_{x} \sigma_{y}} .
$$

To see why this is an ordinary monomer-dimer model, it is useful to take a step backward to a fermionic system: let $\psi_{a}(x)$ and $\bar{\psi}_{a}(x)$ be Grassmann variables associated to the vertex $x$ and $a \in\{1, \ldots, N\}$, and define

$$
\bar{\psi} \psi(x)=\sum_{a=1}^{N} \bar{\psi}_{a}(x) \psi_{a}(x)
$$

then

$$
\begin{gathered}
Z_{G}=N !^{|V(G)|} \int \prod_{x \in V(G)} \prod_{a=1}^{N} d \psi_{a}(x) d \bar{\psi}_{a}(x) \\
e^{m_{x} \bar{\psi} \psi(x)} \prod_{i=(x, y) \in B(G)} e^{w_{x y} \bar{\psi} \psi(x) \bar{\psi} \psi(y) .}
\end{gathered}
$$

Now we go back to a complex spin system by the contour integral formula (2.20), but replace each component $\bar{\psi}_{a}(x) \psi_{a}(x)$ by a complex variable $\sigma_{a}(x)$, so that

$$
\begin{gathered}
Z_{G}=N !^{|V(G)|} \oint\left(\prod_{x \in V(G)} \prod_{a=1}^{N} \frac{d \sigma_{a}(x)}{2 \pi i \sigma_{a}(x)^{2}} e^{m_{x} \sigma_{a}(x)}\right) \\
\prod_{i \in B(G)} \prod_{a, b=1}^{N} e^{w_{x y} \sigma_{a}(x) \sigma_{b}(y)} .
\end{gathered}
$$

Define the new graph $\Gamma$ by

$$
V(\Gamma)=V(G) \times\{1, \ldots, N\}
$$

and

$$
B(\Gamma)=\{(x, a),(y, b)):(x, y) \in B(G), a, b \in\{1, \ldots, N\}\}
$$

with edge weights

$$
W_{(x, a)(y, b)}=W_{x y} .
$$

Talking of $a \in\{1, \ldots, N\}$ as labelling the vertical direction of $\Gamma$, this means that $\Gamma$ is a complete graph in vertical direction. Equation (3.12) is a complex spin system on $\Gamma$ with $N=1$ and therefore equivalent to an ordinary monomer-dimer model.

If quadratic terms had been present in $W$, a term $\sigma_{a}(x) \sigma_{b}(y) \sigma_{c}(x) \sigma_{d}(y)$ with $a \neq c, b \neq d$ would have been possible. Such terms represent pairs of dimers which can be interpreted as more complicated polymers on $\Gamma$. A general interaction, e.g. the one for the $U(N)$-system, can be written as

$$
\sum_{M} W_{M} \prod_{y \in M} \sigma_{y}
$$

$M$ summed over subsets of $\Gamma$ containing $x$ with an even number of elements. Unfortunately the strong results for ordinary dimer systems do not carry over to this type of interactions containing larger polymers. 
Mean Field Theory and Exact Results in Low Dimensions

The partition function of the NJL system on $\Lambda$ can be written as

$$
\begin{gathered}
Z_{\Lambda}=\oint\left(\prod_{x \in \Lambda} \frac{d \sigma_{x}}{2 \pi i \sigma_{x}} \sigma_{x}^{-N}\right) e^{N \sum_{x} I_{x}(\sigma)}, \\
I_{x}(\sigma)=2 m_{x} \sigma_{x}+\frac{1}{2} \sum_{|x-y|=1} \sigma_{x} \sigma_{y}=\sigma_{x}\left(2 m+\frac{1}{2} \sum_{y:|y-x|=1} \sigma_{y}\right) .
\end{gathered}
$$

The mass term acts like an external field on $\sigma_{x}$ and so does the sum over the neighbouring spins. The corresponding mean field model is obtained by replacing this varying field produced by the spins around $x$ by a constant one, $M=2 v\left\langle\sigma_{x}\right\rangle_{\mathrm{MF}}$, which reduces the calculation of $\left\langle\sigma_{y}\right\rangle_{\mathrm{MF}}$ to evaluation of a one-dimensional integral, and the usual consistency condition is simply a quadratic equation

$$
2 v\left\langle\sigma_{x}\right\rangle_{\mathrm{MF}}^{2}+2 m\left\langle\sigma_{x}\right\rangle_{\mathrm{MF}}-1=0
$$

The positive solution

$$
\left\langle\sigma_{x}\right\rangle_{\mathrm{MF}}=\frac{\sqrt{m^{2}+2 v}-m}{2 v} \rightarrow \frac{1}{\sqrt{2 v}}
$$

as $m \rightarrow 0$, so chiral symmetry is broken in the mean-field approximation.

It is possible to set up formal expansions in $1 / v$ or $1 / N$ in which the meanfield result appears as the leading term $[34,30]$. The first order in $1 / N$ was calculated by Rossi and Wolff for the $U(N)$-system [30], and it gives a hint at the failure of the mean-field approximation in two dimensions: the $1 / \mathrm{N}$ correction to the order parameter is divergent.

There is, however, no straightforward way to adapt the proof of the Mermin-Wagner-theorem given by Mermin [37] to the case of complex spin systems: the fact that the action is complex prevents the definition of a local scalar product from which Bogoliubov's inequality can be got as a CauchySchwarz inequality. That the situation is more complicated can be seen from the case $N=1, v=1$, which also represents QED in one dimension. The gauge field can be removed by a gauge transformation so that there remains a system of free fermions, and

$$
\langle\bar{\psi} \psi\rangle=\frac{\operatorname{sign}(m)}{\sqrt{1+m^{2}}} .
$$

The monomer-dimer partition function has not been obtained exactly in two dimensions for general monomer activity $m$ but the counting problem to which this question reduces at $m=0$ has been solved. In this limit, the lattice has to be covered completely by dimers since no monomers are available to fill remaining holes (unoccupied lattice sites). If the dimer activity $w_{1}=1$, the partition function is the number of close-packed dimer configurations on the lattice. It has been shown by Kasteleyn [38] and Temperley and Fisher [39, 35 ] that on a two-dimensional square lattice this number can be written as a Pfaffian, that is, one can find an antisymmetric matrix $A_{\Lambda}$ such that

$$
Z_{\Lambda}(0)=\sqrt{\operatorname{det} A_{\Lambda}},
$$


and it is possible to calculate this determinant exactly (one can of course easily represent the partition function by a permanent, but no method seems to be known presently to evaluate large permanents). Kasteleyn [40] has proven that a matrix $A_{A}$ such that (3.22) holds can be found for any planar graph $\Lambda$. To obtain $Z_{\Lambda}(0)$ on the two-dimensional torus (which is not a planar graph), it suffices to calculate four determinants. A very readable presentation of the solution of the close-packed-dimer problem on a square lattice which various boundary conditions is given in [41].

The two-point function of $\bar{\psi} \psi$ in the original system $\left(\mathrm{QED}_{2}\right)$,

$$
\langle\bar{\psi} \psi(0) \bar{\psi} \psi(x)\rangle_{m=0}=\left\langle\sigma_{0} \sigma_{x}\right\rangle_{m=0}=\frac{Z_{\Lambda \mid\{0, x\}}(0)}{Z_{\Lambda}(0)},
$$

can be calculated as a determinant,

$$
\left\langle\sigma_{0} \sigma_{x}\right\rangle=\left(\operatorname{det}\left(1+\Delta A_{\Lambda}^{-1}\right)\right)^{1 / 2},
$$

where $\Delta=A_{\Lambda \backslash\{0, x\}}-A_{\Lambda}$. This was done in 1963 by Fisher and Stephenson [22] and afterwards by Hartwig [42], with the result that

$$
\left\langle\sigma_{0} \sigma_{x}\right\rangle \sim \frac{1}{|x|^{1 / 2}}
$$

for large $|x|$ (if $x$ is in direction of a lattice axis or the diagonal). Repeating the trivial observation that

$$
\langle\bar{\psi} \psi(x)\rangle_{m=0}=\left\langle\sigma_{x}\right\rangle_{m=0}=0
$$

because the (free) boundary conditions are symmetric, this implies

Remark 3.5. In strongly coupled $\mathrm{QED}_{2}$ at $m=0$, the two-point function of $\bar{\psi} \psi$ clusters.

If there is LRO in this system, it can only show up in some more complicated correlation function. It will be shown below that clustering holds in this model for all $m>0$, so that $X \neq 0$ can happen only if the invariant part of the two-point function is discontinuous at $m=0$. While a discontinuity must occur in $\langle\bar{\psi} \psi\rangle$ at a symmetry-breaking phase transition, its presence in an invariant function would be rather weird. Furthermore, we will show that the four-dimensional model exhibits both LRO at $m=0$ and chiral symmetry breaking in the sense of $X \neq 0$. So, occurrence of chiral symmetry breaking and existence of LRO are very likely to be equivalent (even if this has not been rigorously proven yet in general), and we conclude that chiral symmetry remains unbroken in strongly coupled two-dimensional QED with staggered fermions. This result agrees with what one gets from a calculation in the continuum Schwinger model: the two-point function of the model with two flavours clusters at mass zero.

\section{NJL Model: Rigorous Results}

Consider a monomer-dimer system on the graph $G$ with monomer activities $m_{x}$ and edge weights $w_{x y}$. The partition function satisfies a recursion relation $[20,21]$, which implies 
Theorem 3.6. Let $G$ be a graph, $w_{x y} \geqq 0$ for all $(x, y) \in B(G)$ and $\operatorname{Re} m_{x}>0$ for all $x \in V(G)\left(\right.$ or $\operatorname{Re} m_{x}<0$ for all $\left.x \in V(G)\right)$. Then

$$
Z_{G} \neq 0 \text {. }
$$

Proof. See $[20,21]$. The absolute value of the zeros of $Z_{G}(m)$ cannot exceed $2 \sqrt{W}[20,21]$, where

$$
W=\max _{x \in V(G)} \sum_{y \neq x} w_{x y} .
$$

For the NJL model on $\Lambda, W=2 v N$ does not depend on $|\Lambda|$. That the zeros of $Z_{\Lambda}(m)$ stay in a bounded interval even if $\Lambda \rightarrow \infty$ can also be derived from the convergence of the cluster expansion. Let $\mathscr{W}=\mathbb{C} \backslash 2 i[-\sqrt{2 v N}, \sqrt{2 v N}]$.

Definition 3.7. A function $L: \mathbb{Z}^{v} \rightarrow\{0, \ldots, N+1\}$ for which $\operatorname{supp} L=\left\{x \in \mathbb{Z}^{v}\right.$ : $\left.L_{x} \neq 0\right\}$ is finite is called a finite multiindex. The (countable) set of all such $L$ is

$$
\mathscr{L}=\left\{L: L \text { finite multiindex on } \mathbb{Z}^{\nu}\right\},
$$

and

$$
\left\langle\sigma^{L}\right\rangle_{\Lambda}=\left\langle\prod_{x \in \Lambda} \sigma_{x}^{L_{x}}\right\rangle_{\Lambda} .
$$

Theorem 3.8. Let $\langle\cdot\rangle_{A}$ be the expectation value of the NJL model on $\Lambda \subset \mathbb{Z}^{v}$ and $L$ a finite multiindex.

(1) For all $m \in \mathscr{W}$, the thermodynamic limit

$$
\left\langle\sigma^{L}\right\rangle=\lim _{\Lambda \rightarrow \infty}\left\langle\sigma^{L}\right\rangle_{\Lambda}
$$

exists if $\Lambda \rightarrow \infty$ in the sense of Van Hove.

(2) $\left\langle\sigma^{L}\right\rangle$ is an analytic function of $m$ on $\mathscr{W}$.

Proof. By Remark 3.2, the NJL system on $\Lambda$ is an ordinary monomer-dimer system on a graph $\Gamma(\Lambda)$ with vertex set $V(\Gamma(\Lambda))=\Lambda \times\{1, \ldots, N\}$. The proof of the statement for these systems is contained in [20], see also [28].

Corollary 3.9. Let $m \in \mathbb{R}$. A phase transition can occur in a NJL model only if $m=0$. For $m \neq 0$, all correlation functions are analytic in $m$.

Remark 3.10. Despite analyticity for all $m>0$, the expansion in the hopping parameter $\kappa=1 / 2 m$ does not converge for all $m>0$ due to the zeros of the finite-volume partition functions on the imaginary axis. The radius of convergence of the expansion is, however, at least $(2 \sqrt{2 v N})^{-1}$.

Penrose and Lebowitz have first proven that analyticity of the free energy implies clustering in spin systems [43]. The fact that all correlations are analytic in $m$ for $m>0$ makes it easy to show the same for NJL systems.

Theorem 3.11. In the NJL system exponential clustering holds for all $m \in \mathscr{W}$. More specifically, if $w_{1}=1$,

$$
\left|\left\langle\sigma_{x_{1}} \ldots \sigma_{x_{n}}\right\rangle^{T}\right| \leqq C(n) e^{-\kappa(m) \vartheta\left(x_{1}, \ldots, x_{n}\right)},
$$

where $\kappa(m)>0$ for all $m \in \mathscr{W},\langle\cdot\rangle^{T}$ is the truncated expectation value and $\vartheta\left(x_{1}, \ldots, x_{n}\right)$ is the length of a minimal tree on $\left\{x_{1}, \ldots, x_{n}\right\}$. 
Proof. For all $L \in \mathscr{L},\left|\left\langle\sigma^{L}\right\rangle\right| \leqq 1$ (because monomer-dimer systems can also be represented as limits of Ising systems; this inequality will be proven more generally for reflection-positive complex spin systems below), therefore

$$
F(L, m)=\left\langle\sigma^{L}\right\rangle^{T}
$$

(the superscript $T$ again meaning truncation) obeys a bound

$$
|F(L, m)| \leqq C(L),
$$

where $C(L)$ is the number of summands occurring in the definition of the truncated expectation value. $C(L)$ can be bounded by a function of $n=|L|$ alone. Let $\vartheta(L)$ be the length of the minimal tree connecting the sites in $\operatorname{supp} L$, and for $\vartheta(L) \neq 0$ define

then

$$
u_{L}(m)=\frac{1}{\vartheta(L)} \log \frac{|F(L, m)|}{C(L)},
$$

$$
u_{L}(m) \leqq 0 \forall m \in \mathbb{C}, \quad L \in \mathscr{L} .
$$

For all $L, F(L, \cdot)$ is analytic on $\mathscr{W}$, therefore $u_{L}$ is subharmonic [44], so for all $w$ with $|w-z|<r$,

$$
u_{L}(w) \leqq \int_{0}^{2 \pi} P\left(w-z, r e^{i \theta}\right) u_{L}\left(z+r e^{i \theta}\right) d \theta
$$

if $\{w:|w-z| \leqq r\} \subset \mathscr{W}$, where for $|\zeta|>|z|$

$$
P(z, \zeta)=\frac{1}{2 \pi} \frac{|\zeta|^{2}-|z|^{2}}{|\zeta-z|^{2}} \geqq 0
$$

is Poisson's kernel. By the triangle inequality,

$$
P(z, \zeta) \geqq \frac{1}{2 \pi} \frac{|\zeta|-|z|}{|\zeta|+|z|}
$$

Since all $u_{L}$ are nonpositive,

$$
u(m)=\limsup _{L \in \mathscr{L}} u_{L}(m)
$$

exists and is nonpositive.

For $|m|>m_{0}$, the cluster expansion converges and the truncated correlations decay exponentially, $u_{L}(m) \leqq-K(m)<0$ for all $L$, so $u(m) \leqq-K(m)$ $<0$ for all $m$ with $|m|>m_{0}$. Given an arbitrary element $m \in \mathscr{W}$, there exists a disk centered at some $m_{1}, D=\left\{z \in \mathbb{C}:\left|z-m_{1}\right| \leqq r\right\}$, such that $D \cap\{m \in \mathbb{C}$ : $\left.|m|>m_{0}\right\} \neq \emptyset, D \subset \mathscr{W}$, and $m$ is in the interior of $D$. Since $\delta=r-$ $\left|m-m_{1}\right|>0, P\left(m-m_{1}, r e^{i \theta}\right) \geqq \delta / 4 \pi r$, and

$$
u(m)=\limsup _{L \in \mathscr{L}} u_{L}(m) \leqq \frac{\delta}{4 \pi r} \limsup _{L \in \mathscr{L}} \int_{0}^{2 \pi} d \varphi u_{L}\left(m_{1}+r e^{i \varphi}\right) .
$$

Fatou's Lemma yields

$$
u(m) \leqq \frac{\delta}{4 \pi r} \int_{0}^{2 \pi} \lim \sup _{L \in \mathscr{L}} u_{L}\left(m_{1}+r e^{i \varphi}\right) d \varphi=\frac{\delta}{4 \pi r} \int_{0}^{2 \pi} u\left(m_{1}+r e^{i \varphi}\right) d \varphi
$$


which is negative because $u$ is nonpositive on the whole path and strictly negative on its intersection with the region of cluster expansion convergence (which has nonzero measure). $\kappa(m)=-u(m)$.

Remark 3.12. The clustering rate $\kappa(m)$ is an extension of the function $K(m)$ defined on $\left\{m \in \mathbb{C}:|m|>m_{0}\right\}$; it is independent of the number of spins $n$ occurring in the $n$-point-function (3.32) because in the proof the limit superior was taken over all $L \in \mathscr{L}$.

The Ward identities which one can derive by doing space-dependent chiral rotations relate the expectation value of $\bar{\psi} \psi$ directly to its two-point function for staggered fermions,

$$
\langle\bar{\psi} \psi(y)\rangle=-m \sum_{x} \varepsilon(x) \varepsilon(y)\langle\bar{\psi} \psi(x) \bar{\psi} \psi(y)\rangle^{T}
$$

(the truncation can be included because of the alternating signs $\varepsilon(x)$ in the sum). Inserting the exponential decay with rate $\kappa(m)$ into this equation one sees that if chiral symmetry is broken in the sense of $X \neq 0$, the mass gap $\kappa(m)$ must go to zero as the mass vanishes. One may regard this as a weak version of the Goldstone theorem.

\section{Schwinger-Dyson-Equations}

Let $B_{x y}(t)=\exp \left(N W_{x y}(t)\right)$ for all $(x, y) \in \Lambda^{(1)}, F_{x}(z) \approx \exp \left(N f_{x}(z)\right)$ for all $x$, and $L \in \mathscr{L}$ be a finite multiindex. Then for all $x \in \Lambda$,

$$
\frac{N-L_{x}}{N}\left\langle\sigma^{L}\right\rangle_{A}=\left\langle\sigma_{x} f_{x}^{\prime}\left(\sigma_{x}\right) \sigma^{L}\right\rangle_{A}+\sum_{y:|y-x|=1}\left\langle\sigma_{x} \sigma_{y} W_{x y}^{\prime}\left(\sigma_{x} \sigma_{y}\right) \sigma^{L}\right\rangle_{A} \cdot
$$

Proof. If $L_{x}=N$, the right side is zero because $\sigma_{x}^{N+1}$ appears in the numerator of the contour integral. Let $L_{x}<N$, then the integral over $\sigma_{x}$ contains

$$
\sigma_{x}^{L_{x}-N-1}=-\frac{1}{N-L_{x}} \frac{d}{d \sigma_{x}} \sigma_{x}^{-N-L_{x}}
$$

Integration by parts with respect to $\sigma_{x}$ in the numerator of the defining expression for $\left\langle\sigma^{L}\right\rangle_{A}$ yields (3.44).

The case $F_{x}(\sigma)=\exp (2 N m \sigma)$ for all $x$ and $W_{x y}=W$ will be of special importance; the SD equations then read

$$
\frac{N-L_{x}}{N}\left\langle\sigma^{L}\right\rangle_{A}=2 m\left\langle\sigma_{x} \sigma^{L}\right\rangle_{A}+\sum_{y:|y-x|=1}\left\langle\sigma_{x} \sigma_{y} W^{\prime}\left(\sigma_{x} \sigma_{y}\right) \sigma^{L}\right\rangle_{A} .
$$

\section{Reflection Positivity}

The set $\mathcal{O}_{\boldsymbol{A}}$ of all functions which are analytic at zero forms a $\mathbb{C}$-algebra in a natural way. However, to avoid endless but trivial discussions about the path of integration, these functions were replaced by their truncations to polynomials of degree at most $N$ in every $\sigma_{x}$ and accordingly the definition of the algebra of observables can be given as follows. 
Definition 3.13. Let $\Lambda \subset \mathbb{Z}^{v}$ finite, $\mathscr{L}(\Lambda)=\{L \in \mathscr{L} \mid \operatorname{supp} L \subset \Lambda\}$, and

$$
\mathscr{A}_{\Lambda}=\left\{A: \mathbb{C}^{\Lambda} \rightarrow \mathbb{C}, \sigma \mapsto A(\sigma) \mid A(\sigma)=\sum_{L \in \mathscr{L}(\Lambda)} a_{L} \sigma^{L}, a_{L} \in \mathbb{C}\right\}
$$

For $L, L^{\prime} \in \mathscr{L}(\Lambda), L+L^{\prime} \in \mathscr{L}(\Lambda)$ is defined by

$$
\left(L+L^{\prime}\right)_{x}=\min \left\{L_{x}+L_{x}^{\prime}, N+1\right\},
$$

for $A, B \in \mathscr{A}_{\Lambda}, A(\sigma)=\sum a_{L} \sigma^{L}$ and $B(\sigma)=\sum b_{L} \sigma^{L}, A B \in \mathscr{A}_{\Lambda}$ is defined as

$$
(A B)(\sigma)=\sum_{L, L^{\prime} \in \mathscr{L}(\Lambda)} a_{L} b_{L^{\prime}} \sigma^{L+L^{\prime}}
$$

It is obvious that with this definition $\mathscr{A}_{\Lambda}$ is a $\mathbb{C}$-algebra.

For $\mu \in\{1, \ldots, v\}$ the reflection $r_{\mu}$ is given by $\left(r_{\mu} x\right)_{\lambda}=x_{\lambda}$ for $\lambda \neq \mu$, and $\left(r_{\mu} x\right)_{\mu}=L_{\mu}-1-x_{\mu}$. The reflection plane $E_{\mu}=\left\{y \in \mathbb{R}^{v} \mid y_{\mu}=\left(L_{\mu}-1\right) / 2\right\}$ lies between the planes containing lattice sites, it cuts the lattice into two halves, $\Lambda=\Lambda_{+} \cup \Lambda_{-}$, with $\Lambda_{+}=\left\{x \in \Lambda \mid x_{\mu} \geqq \frac{L_{\mu}-1}{2}\right\}$ and $\Lambda_{-}=r_{\mu} \Lambda_{+} \cdot$ If $\Lambda$ is a torus, not only the links $\left(x, x+e_{\mu}\right)$ with $x_{\mu}=\frac{L_{\mu}}{2}-1$ but also those with $x_{\mu}=L_{\mu}-1$ connect $\Lambda_{+}$with $\Lambda_{-}$. For simplicity of notation, in the following the reflection $r_{1}$ will be considered and called $r$.

Definition 3.14. Let $L \in \mathscr{L}(\Lambda)$. The reflection on the field algebra corresponding to $r$ is the antilinear map $\Theta: \mathscr{A}_{\Lambda} \rightarrow \mathscr{A}_{\Lambda}$ with

$$
(\Theta A)(\sigma)=\sum_{L \in \mathscr{L}(\Lambda)} \bar{a}_{L} \sigma^{L_{r}}
$$

for $A=\sum a_{L} \sigma^{L}$, where $L_{r}(x)=L(r x)$. The complex spin system on $\Lambda$ is called reflection positive (RP) if for all $A \in \mathscr{A}_{\Lambda_{+}}$,

$$
\langle A \Theta A\rangle_{\Lambda} \geqq 0 .
$$

Proposition 3.15. Let $F_{x}(\sigma)=F(\sigma)$ for all $x \in \Lambda$, where

$$
F(\bar{\sigma})=\overline{F(\sigma)}
$$

and

$$
B(t)=\sum_{k=0}^{N} a_{k} t^{k}
$$

If $a_{k} \geqq 0$ for all $k \in\{0, \ldots, N\}$ then the complex spin system with $F$ and $B$, as given by Definition 3.1, is RP,

Proof. As in [45, 46]. The condition $a_{k} \geqq 0$ is used when the terms coupling $\Lambda_{+}$and $\Lambda_{-}$are considered.

Remark 3.16. RP implies that $\langle A, B\rangle=\langle A \Theta B\rangle_{A}$ is a semiscalar product on $\mathscr{A}_{A_{+}}$and therefore the Schwarz inequality

$$
|\langle A \Theta B\rangle|^{2} \leqq\langle A \Theta A\rangle\langle B \Theta B\rangle
$$


holds for all $A, B \in \mathscr{A}_{\Lambda_{+}}$. In particular, for all $x$,

$$
\left|\left\langle\sigma_{x}\right\rangle\right|^{2}=\left|\left\langle\sigma_{x} \Theta 1\right\rangle\right|^{2} \leqq\left\langle\sigma_{x} \sigma_{r x}\right\rangle
$$

since $\langle 1 \Theta 1\rangle=1$. Let $x_{1}=L_{\mu} / 2$, then $r x=x-e_{1}$ and

$$
\left|\left\langle\sigma_{x}\right\rangle\right|^{2} \leqq\left\langle\sigma_{x} \sigma_{x-e_{1}}\right\rangle \text {. }
$$

Let $\varepsilon(x)$ be given by (2.8), $\Lambda_{e}=\{x \in \Lambda \mid \varepsilon(x)=1\}$, and $\Lambda_{o}=\Lambda \backslash \Lambda_{e}$. As proven in [19], reflection positivity implies the

Theorem 3.17 (Chessboard bounds). Let $G_{x}: \mathbb{C} \rightarrow \mathbb{C}$ be analytic at zero for all $x \in \Lambda$, and $\langle\cdot\rangle_{\Lambda} \mathrm{RP}$, then

$$
\left|\left\langle\prod_{x \in \Lambda} G_{x}\left(\sigma_{x}\right)\right\rangle_{\Lambda}\right| \leqq \prod_{y \in \Lambda}\left\langle\prod_{x \in \Lambda_{e}} G_{y}\left(\sigma_{x}\right) \prod_{x \in \Lambda_{o}} \bar{G}_{y}\left(\bar{\sigma}_{x}\right)\right\rangle^{\frac{1}{|\Lambda|}} .
$$

Theorem 3.18. Let $N \in \mathbb{N}, F_{x}(z)=e^{2 N m z}$ for all $x \in \Lambda$, and $B_{x y}(t)=e^{N W(t)}$ for all $(x, y) \in \Lambda^{(1)}$ with

$$
W(t)=\sum_{k=1}^{N} w_{k} t^{k}
$$

$w_{1}=1$ and $w_{k} \geqq 0$ for all $k \in\{2, \ldots, N\}$, and $\langle\cdot\rangle_{\Lambda}$ the expectation value of the corresponding complex spin system on $\Lambda$. Then

(1) For all $\Lambda, N, m \geqq 0$ and $L \in \mathscr{L}$,

$$
0 \leqq\left\langle\sigma^{L}\right\rangle_{\Lambda} \leqq 1
$$

(2) For all $N, m \in \mathbb{R}$, thermodynamic limits

$$
\lim _{\Lambda \rightarrow \infty}\left\langle\sigma^{L}\right\rangle_{\Lambda}=\left\langle\sigma^{L}\right\rangle
$$

exist and obey (3.59).

(3) The Fourier transforms of $\left\langle\sigma^{L}\right\rangle$ are distributions on $\mathscr{B}_{v}=[-\pi, \pi]^{v}$.

Proof. (1) That $\left\langle\sigma^{L}\right\rangle \geqq 0$ is clear from the monomer-dimer representation because $m \geqq 0$ and all $w_{k} \geqq 0$. All terms on the right side of the SD equation (3.46) are of the form $a\left\langle\sigma^{L}\right\rangle$ with nonnegative $a$, hence nonnegative. Since $w_{1}=1,(3.46)$ reads

$$
\frac{N-L_{x}}{N}\left\langle\sigma^{L}\right\rangle_{\Lambda}=2 m\left\langle\sigma_{x} \sigma^{L}\right\rangle_{\Lambda}+\sum_{y:|x-y|=1}\left\langle\sigma_{x} \sigma_{y} \sigma^{L}\right\rangle_{\Lambda}+\varrho
$$

with $\varrho \geqq 0$. Dropping $\varrho$, the term multiplied by $m$ and all terms in the sum over $y$ except for the one with $y=x+e_{1}$,

$$
\left\langle\sigma_{x} \sigma_{x+e_{1}} \sigma^{L}\right\rangle_{\Lambda} \leqq \frac{N-L_{x}}{N}\left\langle\sigma^{L}\right\rangle_{\Lambda} \leqq\left\langle\sigma^{L}\right\rangle_{\Lambda}
$$

Since

$$
\Lambda=\bigcup_{x \in \Lambda_{e}}\left\{x, x+e_{1}\right\}
$$


iteration of (3.62) yields

$$
\left\langle\prod_{x \in \Lambda} \sigma_{x}^{l+1}\right\rangle_{\Lambda}=\left\langle\prod_{x \in \Lambda} \sigma_{x}^{l} \prod_{x \in A_{e}} \sigma_{x} \sigma_{x+e_{1}}\right\rangle_{\Lambda} \leqq\left\langle\prod_{x \in \Lambda} \sigma_{x}^{l}\right\rangle_{\Lambda} \leqq \cdots \leqq\langle 1\rangle_{\Lambda}=1 .
$$

Given $L \in \mathscr{L}$, set $G_{x}\left(\sigma_{x}\right)=\sigma_{x}^{L_{x}}=\overline{G_{x}\left(\bar{\sigma}_{x}\right)}$ and apply the chessboard bound (3.57) to get

$$
\left\langle\sigma^{L}\right\rangle_{\Lambda} \leqq \prod_{y \in \Lambda}\left\langle\prod_{x \in \Lambda} \sigma_{x}^{L_{y}}\right\rangle^{\frac{1}{|\Lambda|}} \leqq 1 .
$$

(2) Let $X=L^{1}(\mathscr{L}, \mathbb{C})$ and

$$
\zeta=\left\{\Lambda \subset \mathbb{Z}^{v}:|\Lambda|<\infty\right\} .
$$

For $\Lambda \in \zeta$, define the linear functional

$$
f_{\Lambda}:\left\{\begin{array}{l}
X \rightarrow \mathbb{C} \\
a=\left(a_{L}\right)_{L \in \mathscr{L}} \mapsto f_{\Lambda}(a)=\sum_{L \in \mathscr{L}} a_{L}\left\langle\sigma^{L}\right\rangle_{\Lambda} .
\end{array}\right.
$$

By (1), for all $a \in X$ and all $m \in \mathbb{R}\left(m \rightarrow-m\right.$ only changes the sign of $\left.\left\langle\sigma^{L}\right\rangle\right)$,

$$
\left|f_{\Lambda}(a)\right| \leqq \sum_{L \in \mathscr{L}}\left|a_{L}\right|=\|a\|,
$$

so $f_{A} \in X^{*}$ and $\left\|f_{A}\right\| \leqq 1$. By the Banach-Alaoglu-theorem [47], the sequence $\left(f_{A}\right)_{\Lambda \in \zeta}$ has accumulation points. By definition of weak-*-convergence, there are subsequences $\left(\Lambda_{k}\right)_{k \in \mathbb{N}}$ so that $\left(f_{\Lambda_{k}}(a)\right)_{k \in \mathbb{N}}$ converges for all $a \in X$.

Remark 3.19. (1) For the NJL models $\left(w_{k}=0\right.$ for $\left.k \geqq 2\right)$ and $m>0$, existence, analyticity and uniqueness of the thermodynamic limit have already been proven before. So, if $\Lambda \rightarrow \infty$ in the sense of Van Hove, the limit coincides with the one constructed earlier. Theorem 3.18 ensures existence of a thermodynamic limit also for $m=0$, but not, of course, uniqueness.

(2) The results obtained so far do not show that in the infinite-volumelimit

$$
\lim _{m \rightarrow 0}\left\langle\sigma_{x}\right\rangle \propto \lim _{m \rightarrow 0}\langle\bar{\psi} \psi\rangle
$$

exists. However, because of the boundedness (3.59), limsup and lim inf do, and therefore the order parameters defined above exist.

Theorem 3.20. Let $\langle\cdot\rangle$ RP. For all $A, B, C_{i}, D_{i} \in \mathscr{A}_{A_{+}}$,

$$
\left|\left\langle e^{A+\Theta B+\Sigma C_{i} \Theta D_{i}}\right\rangle\right|^{2} \leqq\left\langle e^{A+\Theta A+\Sigma C_{i} \Theta C_{i}}\right\rangle\left\langle e^{B+\Theta B+\Sigma D_{i} \Theta D_{i}}\right\rangle .
$$

Proof. See [19].

Theorem 3.21 (Infrared Bound). Let $\Lambda$ be a torus,

$$
\begin{aligned}
F_{x}(z) & =F(z)=\overline{F(\bar{z})} \forall x \in \Lambda, \\
B_{x y}(t) & =\exp N W(t) \forall(x, y) \in \Lambda^{(1)},
\end{aligned}
$$

with

$$
W(t)=\sum_{k=1}^{N} w_{k} t^{k}
$$


$V(t)=W(t)-t$, and define $b_{k} b y$

$$
e^{N V(t)}=\sum_{k \geqq 0} b_{k} t^{k}
$$

If $b_{k} \geqq 0$ for all $k \in\{0, \ldots, N\}$, then for all $h: \Lambda \rightarrow \mathbb{R}$,

$$
\langle\sigma(h) \sigma(-\Delta h)\rangle_{\Lambda} \leqq \frac{1}{N}\|h\|^{2}
$$

and

$$
\langle\sigma(h) \sigma(\bar{\Delta} h)\rangle_{\Lambda} \geqq-\frac{1}{N}\|h\|^{2} .
$$

$\Delta$ is the Laplacean, $\bar{\Delta}$ its antiferromagnetic counterpart, defined by

$$
(\bar{\Delta} f)(x)=\sum_{\mu}\left(f\left(x+e_{\mu}\right)+f\left(x-e_{\mu}\right)+2 f(x)\right),
$$

and

$$
\sigma(h)=(\sigma, h)=\sum_{x \in \Lambda} \sigma_{x} h_{x}, \quad\|h\|^{2}=(h, h)
$$

Proof. Define an unnormalized background expectation value $[\cdot]_{\Lambda}$ by

$$
[f]_{\Lambda}=\oint \prod_{x \in \Lambda} \frac{d \sigma_{x}}{2 \pi i \sigma_{x}} \sigma_{x}^{-N} F\left(\sigma_{x}\right) e^{\varepsilon N v \sigma_{x}^{2}} \prod_{x, y} e^{N V\left(\sigma_{x} \sigma_{x}+e_{\mu}\right)} f(\sigma) .
$$

By Proposition 3.15, $[\cdot]_{\Lambda}$ is RP with respect to the reflection $\Theta$ given in Definition 3.14. For $\varphi: \Lambda \rightarrow \mathbb{C}$ and $\varepsilon \in\{-1,1\} \quad(\varepsilon=-1$ is needed for the lower bound), let

$$
H_{\Lambda}^{\varepsilon}(\varphi)=\frac{\varepsilon}{2} \sum_{x, y}\left(\left(\sigma_{x}-\varphi_{x}\right)-\varepsilon\left(\sigma_{x+e_{\mu}}-\varphi_{x+e_{\mu}}\right)\right)^{2}
$$

and

$$
Z_{\Lambda}^{\varepsilon}(\varphi)=\left[e^{-N H_{\Lambda}(\varphi)}\right]_{\Lambda},
$$

then $Z_{\Lambda}^{\varepsilon}(0)=Z_{\Lambda}$ and

$$
\begin{aligned}
Z_{\Lambda}^{\varepsilon}(\varphi)= & Z_{\Lambda} \exp \left(-\frac{\varepsilon N}{2} \sum_{x, \mu}\left(\varphi_{x}-\varepsilon \varphi_{x+e_{\mu}}\right)^{2}\right) \\
& \cdot\left\langle\prod_{x, \mu} \exp \left(\varepsilon N\left(\sigma_{x}-\varepsilon \sigma_{x+e_{\mu}}\right)\left(\varphi_{x}-\varepsilon \varphi_{x+e_{\mu}}\right)\right)\right\rangle_{\Lambda},
\end{aligned}
$$

i.e.

$$
Z_{\Lambda}^{+}(\varphi)=e^{-\frac{N}{2}(\varphi,-\Delta \varphi)} Z_{\Lambda}\left\langle e^{N(\sigma,-\Delta \varphi)}\right\rangle_{\Lambda}
$$

and

$$
Z_{\bar{\Lambda}}^{-}(\varphi)=e^{\frac{N}{2}(\varphi, \bar{\Delta} \varphi)} Z_{\Lambda}\left\langle e^{-N(\sigma, \bar{\Delta} \varphi)}\right\rangle_{\Lambda}
$$

$H_{\Lambda}^{\varepsilon}$ can be rewritten as

where

$$
H_{\Lambda}^{\varepsilon}(\varphi)=H_{\Lambda_{+}}^{\varepsilon}(\varphi)+\Theta H_{\Lambda_{+}}^{\varepsilon}(\bar{\varphi} \circ r)+H_{C}(\varphi),
$$

$$
H_{\Lambda}^{\varepsilon}(\varphi)=\varepsilon v \sum_{x \in \Lambda_{+}}\left(\sigma_{x}-\varphi_{x}\right)^{2}-\sum_{x, \mu}\left(\sigma_{x}-\varphi_{x}\right)\left(\sigma_{x+e_{\mu}}-\varphi_{x+e_{\mu}}\right),
$$


[the second sum is understood to contain only $(x, \mu)$ for which both $x$ and $x+e_{\mu}$ are in $\left.\Lambda_{+}\right]$and

$$
H_{C}(\varphi)=-\sum_{x: x_{1} \in\left\{0, L_{1}\right\}}\left(\sigma_{x}-\varphi_{x}\right)\left(\sigma_{x-e_{1}}-\varphi_{x-e_{1}}\right)
$$

consists of the terms coupling $\Lambda_{+}$and $\Lambda_{-}$,

$$
\begin{gathered}
H_{C}(\varphi)=-\sum_{x: x_{1} \in\left\{0, L_{1}\right\}} C_{x} \Theta D_{x}, \\
C_{x}=\sigma_{x}-\varphi_{x}, \\
D_{x}=\sigma_{x}-\bar{\varphi}_{r x} .
\end{gathered}
$$

Denoting $\Lambda_{l}=\left\{x \in \Lambda: x_{1}=l\right\}$ and $\varphi^{(l)}=\left(\varphi_{x}\right)_{x \in \Lambda_{l}} \in \mathbb{C}^{\Lambda_{l}}$,

$$
F_{\varepsilon}\left(\varphi^{(0)}, \ldots, \varphi^{\left(L_{1}-1\right)}\right)=Z_{\Lambda}^{\varepsilon}(\varphi)
$$

defines a function $F: \mathbb{C}^{\Lambda_{1}} \times \cdots \times \mathbb{C}^{\Lambda_{1}} \rightarrow \mathbb{C}$ which fulfills cyclicity due to the periodic boundary conditions on $\Lambda$. Using Theorem 3.20 which is applicable because $[\cdot]_{A}$ is reflection-positive (normalization does not matter), one sees that

$$
\begin{gathered}
\left|F_{\varepsilon}\left(\varphi^{(0)}, \ldots, \varphi^{\left(L_{1}-1\right)}\right)\right|^{2} \leqq F_{\varepsilon}\left(\varphi^{(0)}, \ldots, \varphi^{\left(\frac{L_{1}}{2}-1\right)}, \bar{\varphi}^{\left(\frac{L_{1}}{2}-1\right)}, \ldots, \bar{\varphi}^{(0)}\right) \\
F_{\varepsilon}\left(\bar{\varphi}^{\left(L_{1}-1\right)}, \ldots, \bar{\varphi}^{\left(\frac{L_{1}}{2}-1\right)}, \varphi^{\left(\frac{L_{1}}{2}-1\right)}, \ldots, \varphi^{\left(L_{1}-1\right)}\right),
\end{gathered}
$$

so the abstract chessboard bounds [19] apply (with the involution defined as complex conjugation) and

$$
\left|Z_{\Lambda}^{\varepsilon}(\varphi)\right| \leqq \prod_{l=0}^{L_{1}-1} F_{\varepsilon}\left(\varphi^{(l)}, \bar{\varphi}^{(l)}, \ldots, \varphi^{(l)}, \bar{\varphi}^{(l)}\right)^{\frac{1}{L_{1}}} .
$$

The factors on the right side are partition functions $Z_{\Lambda}^{\varepsilon}(\tilde{\varphi})$ with $\tilde{\varphi}$ consisting of identical or reflected copies of $\varphi^{(l)}$ in each plane $\Lambda_{l}$. Repetition of this argument for all other directions implies that

$$
\left|Z_{\Lambda}^{\varepsilon}(\varphi)\right| \leqq \prod_{x \in \Lambda} Z_{\Lambda}^{\varepsilon}\left(\Phi^{(x)}\right)^{\frac{1}{|\Lambda|}}
$$

where

$$
\Phi_{y}^{(x)}=\left\{\begin{array}{ll}
\varphi_{x} & \text { if } y \in \Lambda_{e} \\
\bar{\varphi}_{x} & \text { if } y \in \Lambda_{0}
\end{array} .\right.
$$

For $\varepsilon=1$, take $\varphi_{x} \in \mathbb{R}$ for all $x$, then $\Phi^{(x)}$ is a constant configuration, and

$$
H_{\Lambda}^{+}\left(\Phi^{(x)}\right)=H_{A}^{+}(0)
$$

that is, $Z_{\Lambda}^{+}\left(\Phi^{(x)}\right)=Z_{\Lambda}$ and consequently Gaussian domination $[18,19]$

$$
\left|Z_{\Lambda}^{+}(\varphi)\right| \leqq Z_{\Lambda}
$$

holds. For $\varepsilon=-1$, take $\varphi_{x} \in i \mathbb{R}$, then $\Phi^{(x)}$ is a staggered configuration. Since $H_{\Lambda}^{-}$is formally antiferromagnetic,

$$
Z_{\Lambda}^{-}\left(\Phi^{(x)}\right)=Z_{\Lambda}
$$

and thus

$$
\left|Z_{\Lambda}^{-}(\varphi)\right| \leqq Z_{\Lambda}
$$


Together with (3.82) and (3.83), this means that for all $\varphi: \Lambda \rightarrow \mathbb{R}$,

$$
\left|\left\langle e_{\Lambda}^{N(\sigma,-\Delta \varphi)}\right\rangle\right| \leqq e^{\frac{N}{2}(\varphi,-\Delta \varphi)}
$$

and

$$
\left|\left\langle e_{\bar{\Lambda}}^{-i N(\sigma, \bar{\Delta} \varphi)}\right\rangle\right| \leqq e^{\frac{N}{2}(\varphi, \bar{\triangle} \varphi)} .
$$

The rest of the proof is similar to the one in [18].

Remark 3.22. For the NJL model $V(t)=0$ and the infrared bound can also be proven by application of the chessboard bounds along the lines of [19].

Let

$$
T_{A}(x)=\left\langle\sigma_{y} \sigma_{y+x}\right\rangle_{A}=T_{A}(-x)
$$

be the two-point function. At $m=0$, because of chiral symmetry, $\left\langle\sigma_{0} \sigma_{x}\right\rangle_{A}=0$ if $\varepsilon(x) \varepsilon(0)=1$, so $($ since $\varepsilon(x)=\exp (i \hat{\pi} x)$ with $\hat{\pi}=(\pi, \ldots, \pi))$

$$
T_{\Lambda}(x)=\frac{1}{2} T_{\Lambda}(x)\left(1-e^{i \hat{\pi} x}\right) \text { for } m=0 .
$$

For $\varphi: \Lambda \rightarrow \mathbb{C}$, define

$$
T_{\Lambda}(\varphi)=\left(T_{\Lambda}, \varphi\right)=\sum_{x \in A} T_{\Lambda}(x) \varphi_{x}
$$

and $\hat{T}_{A}$ by

$$
\hat{T}_{\Lambda}(\hat{\varphi})=T_{\Lambda}(\varphi),
$$

then

$$
\hat{T}_{\Lambda}(\hat{\varphi})=\frac{1}{|\Lambda|} \sum_{k \in \Lambda^{*}} \hat{T}_{\Lambda}(k) \hat{\varphi}_{k}
$$

with

$$
\hat{T}_{\Lambda}(k)=\sum_{x \in \Lambda} e^{-i k x} T_{\Lambda}(x),
$$

and

$$
\hat{T}_{\Lambda}(k+\hat{\pi})=-\hat{T}_{\Lambda}(k) \forall k \in \Lambda^{*} \text { if } m=0 .
$$

Theorem 3.23. (1) Let $v \geqq 3, m \in \mathbb{R}, F_{x}(z)=e^{2 N m z}$ for all $x \in \Lambda$, and the complex spin system on $A$ fulfill the conditions of Theorem 3.21, and assume that the two-point function $T_{A}(x)$ satisfies $\left|T_{A}(x)\right| \leqq 1$ for all $x$. Any thermodynamic limit of the two-point function

$$
\hat{T}=\lim _{\Lambda \rightarrow \infty} \hat{T}_{\Lambda}
$$

is a signed measure on $\mathscr{B}_{v}=[-\pi, \pi]^{v}$.

$$
\hat{T}(k)=c_{0} \delta(k)-c_{\hat{\pi}} \delta(k-\hat{\pi})+\hat{g}(k)
$$

with $c_{0} \geqq 0, c_{\hat{\pi}} \geqq 0\left(c_{0}=c_{\hat{\pi}}\right.$ at $\left.m=0\right), \hat{g}$ absolutely continuous with respect to Lebesgue measure, and

$$
-\frac{1}{2 N(2 \pi)^{v} D(k+\hat{\pi})} \leqq \hat{g}(k) \leqq \frac{1}{2 N(2 \pi)^{v} D(k)}
$$


with the kernel of the Laplacean

$$
2 D(k)=2 \sum_{\mu=1}^{v}\left(1-\cos k_{\mu}\right)
$$

(2) For $v \geqq 3$, in the thermodynamic limit of the NJL model at $m \neq 0$,

$$
\hat{T}(k)=s^{2} \delta(k)+\hat{g}(k),
$$

where $s=\left\langle\sigma_{x}\right\rangle, \hat{g}$ is analytic for $\left|\operatorname{Im} k_{\mu}\right|<\kappa(m)$, and $\hat{g}$ obeys the bound (3.109). Proof. Upon Fourier transformation, (3.74) and (3.75) become

$$
\begin{gathered}
2 \sum_{k \in \Lambda^{*}} \hat{T}_{\Lambda}(k) D(k)\left|\hat{h}_{k}\right|^{2} \leqq N^{-1} \sum_{k \in \Lambda^{*}}\left|\hat{h}_{k}\right|^{2} \\
2 \sum_{k \in \Lambda^{*}} \hat{T}_{\Lambda}(k) D(k+\hat{\pi})\left|\hat{h}_{k}\right|^{2} \geqq-N^{-1} \sum_{k \in \Lambda^{*}}\left|\hat{h}_{k}\right|^{2},
\end{gathered}
$$

for all $\hat{h}$ with $\hat{h}_{-k}=\overline{\hat{h}_{k}} . D(k+\hat{\pi})$ is the kernel of the antiferromagnetic Laplacean in momentum space. The constraint on $\hat{h}$ does not matter since $D(k)=D(-k)$ and $\hat{T}_{\Lambda}(-k)=\hat{T}_{\Lambda}(k)$; choosing $\hat{h}_{k}=\delta_{k, k_{0}}+\delta_{k,-k_{0}}$, we have for all $k \in \Lambda^{*} \backslash\{0, \hat{\pi}\}$,

$$
\left|\hat{T}_{\Lambda}(k)\right| \leqq \frac{1}{2 N} b(k)
$$

with

$$
b(k)=\sup \left\{D(k)^{-1}, D(k+\hat{\pi})^{-1}\right\} .
$$

In the thermodynamic limit, $\Lambda^{*}=\mathscr{B}_{v}$. For $v \geqq 3, b \in L^{1}\left(\mathscr{B}_{v}\right)$.

$\hat{T}_{A}$ can be viewed as a distribution on $C^{\infty}\left(\mathscr{B}_{v}\right)$,

$$
\hat{T}_{\Lambda}=\frac{1}{|\Lambda|} \sum_{k \in \Lambda^{*}} \hat{T}_{\Lambda}(k) \delta_{k}
$$

$\left(\delta_{k}\right.$ is Dirac's delta located at $\left.k\right)$. Let $\varphi \in C^{\infty}\left(\mathscr{B}_{v}\right)$, then

$$
\begin{aligned}
\left|\hat{T}_{\Lambda}(\varphi)\right| & \leqq \frac{1}{|\Lambda|} \sum_{k \in \Lambda^{*}}|\varphi(k)|\left|\hat{T}_{\Lambda}(k)\right| \\
& \leqq\|\varphi\|_{\infty}\left(\frac{1}{|\Lambda|} \sum_{k \notin\{0, \hat{\pi}\}} b(k)+\frac{1}{|\Lambda|}\left(\left|\hat{T}_{\Lambda}(0)\right|+\left|\hat{T}_{\Lambda}(\hat{\pi})\right|\right)\right) .
\end{aligned}
$$

Since $T_{A}$ is bounded,

$$
\frac{1}{|\Lambda|}\left|\hat{T}_{\Lambda}(p)\right| \leqq \frac{1}{|\Lambda|} \sum_{x \in \Lambda}\left|T_{\Lambda}(x)\right| \leqq 1 .
$$

Applying this to $p=0$ and $p=\hat{\pi}$,

$$
\left|\hat{T}_{\Lambda}(\varphi)\right| \leqq\|\varphi\|_{\infty}\left(2+\frac{1}{2 N|\Lambda|} \sum_{k \in \Lambda \backslash\{0, \hat{\pi}\}} b(k)\right),
$$

and because $b$ is integrable, the sum converges to $(2 \pi)^{-v}\|b\|_{1}$ and in the limit

$$
\lim _{\Lambda \rightarrow \infty}\left|\hat{T}_{\Lambda}(\varphi)\right|=|\hat{T}(\varphi)| \leqq\left(2+\frac{1}{2 N(2 \pi)^{v}}\|b\|_{1}\right)\|\varphi\|_{\infty},
$$


so $\hat{T}$ is a signed measured by the Riesz-Markov-theorem [47]. Inequality (3.109) follows by taking the thermodynamic limit in the infrared bound and using a peaking argument, as in [18].

(2) By Theorem 3.11, $\left\langle\sigma_{0} \sigma_{x}\right\rangle-\left\langle\sigma_{0}\right\rangle\left\langle\sigma_{x}\right\rangle=g(x)$ clusters exponentially for $m \neq 0$, therefore its Fourier transform $\hat{g}$ is analytic in $k$ for $\left|\operatorname{Im} k_{\mu}\right|<\kappa(m)$. The $\delta$-term in $\hat{T}$ is the Fourier transform of the constant $s^{2}=\left\langle\sigma_{0}\right\rangle\left\langle\sigma_{x}\right\rangle$.

\section{Chiral Symmetry Breaking}

This section contains the results of the analysis of complex spin systems done above. That the mean-field solution is an upper bound for the order parameter is a simple consequence of reflection positivity and the Schwinger-Dyson equations (SDE). The strategy of the proof of chiral symmetry breaking is the same as the one used by Fröhlich, Simon, and Spencer [18] to prove the existence of long-range order in continuous spin models at low temperature, namely to combine a lower bound for the two-point function at a fixed distance with the infrared bound for its Fourier transform. In the case of the $O(N)$-model, the lower bound is provided by $\left\langle s_{x} \cdot s_{x}\right\rangle=\langle 1\rangle=1$ simply because the length of the spin vector is fixed. In the complex spin systems derived from the strongly coupled fermion-gauge systems there is no simple positive lower bound for $\left\langle\sigma_{x}^{2}\right\rangle \propto\left\langle\bar{\psi} \psi(x)^{2}\right\rangle$ independently of $m$ because this function is not invariant (in the case $N=1$ it is even zero because of nilpotency, and for $N>1$ the proof of a positive lower bound for this function is essentially the same problem as the proof of a lower bound for $\left\langle\sigma_{x}\right\rangle$ itself. However, the SDE (3.46) can be used to give a lower bound for $\left\langle\sigma_{x} \sigma_{x+e_{\mu}}\right\rangle$, especially $\left\langle\sigma_{x} \sigma_{x+e_{\mu}}\right\rangle=1 / 2 v$ for all $\mu$ at $m=0$, and this lower bound can be used for a proof of chiral symmetry breaking.

The fact that clustering could be shown for the NJL models allows to prove a lower bound for the chiral order parameter $X$, more generally, the SDE and the infrared bound combine nicely to a lower bound for $\langle\bar{\psi} \psi\rangle$ as a function of $m$ which is of exactly the same form as the upper bound by the mean field solution except that in it the fluctuations are controlled instead of neglected. Their effect on the order parameter can be estimated by a function $S$ which depends on the dimension. $S(v)$ is not the integral occurring in the $O(N)$-models [18] (which is denoted $R$ in the appendix) because the integrand contains an additional cosine in the numerator due to the fact that $\left\langle\sigma_{x} \sigma_{x+e_{\mu}}\right\rangle$ is used to get the lower bound. So let us start with the definition of $S$ and a list of its properties.

Definition 4.1.

$$
\begin{gathered}
C(k)=\sum_{\mu=1}^{v} \cos k_{\mu}=v-D(k), \\
\mathscr{B}_{v}^{+}=\left\{k \in \mathscr{B}_{\nu} \mid C(k)>0\right\},
\end{gathered}
$$

for $v \geqq 3$

$$
S(v)=\int_{\delta_{v}^{+}} \frac{d^{v} k}{(2 \pi)^{v}} \frac{C(k)}{D(k)} .
$$


Proposition 4.2. (1) $0<S(v)<\infty$ for all $v \geqq 3$.

(2) Let $R(v)$ be given by (A.4). Then

$$
S(v) \leqq v R(v)-\frac{3}{4} \leqq \frac{v}{v-2}-\frac{3}{4},
$$

and both upper bounds are decreasing functions of $v$.

(3) $S(v) \rightarrow 0$ as $v \rightarrow \infty$, for $v \geqq v_{0}$

$$
|S(v)| \leqq c\left(v_{0}\right) v^{-1 / 4} .
$$

(4) $S(3)<0.64, S(4)<0.35, S(5)<0.26, S(v) \leqq S(4)$ for all $v \geqq 4$.

Proof. See Appendix.

Chiral Symmetry Breaking in QED and the NJL Model

Let $N \in \mathbb{N}$, and $\langle\cdot\rangle_{A}$ denote the expectation value of the NJL model on $\Lambda$ as defined in (3) of Definition 3.3. Recall that $\langle\bar{\psi} \psi(x)\rangle=2 N\left\langle\sigma_{x}\right\rangle$. Without loss of generality, $m \geqq 0$. By Theorem 3.18 the thermodynamic limit of the correlation functions exists, they are unique and analytic in $m$ if $m \neq 0$ by Theorem 3.8, and exponential clustering holds for $m \neq 0$ (Theorem 3.11). The infrared bound implies the special properties of the two-point function stated in Theorem 3.23.

Theorem 4.3. For all $m \geqq 0$ and all $v \geqq 1$,

$$
\left\langle\sigma_{x}\right\rangle \leqq s_{1},
$$

where $s_{1}$ is the positive solution (3.20) of the mean-field equation

$$
2 v s_{1}^{2}+2 m s_{1}-1=0
$$

If $v \geqq 3$ and $\frac{2}{N} S(v)<1$, for all $m>0$

$$
\left\langle\sigma_{x}\right\rangle \geqq s_{2},
$$

where $s_{1}$ is the positive solution (3.20) of the mean-field equation

$$
2 v s_{2}^{2}+2 m s_{2}-\left(1-\frac{2}{N} S(v)\right)=0
$$

Proof. Consider the SD equation (3.46) in infinite volume for $L=0$,

$$
1-2 m\left\langle\sigma_{x}\right\rangle=\sum_{y:|y-x|=1}\left\langle\sigma_{x} \sigma_{y}\right\rangle .
$$

Using the lattice rotational symmetry of the two-point function which holds because the thermodynamic limit is unique for $m>0$ and the symmetry holds in the cluster expansion region, this becomes

$$
1=2 m\left\langle\sigma_{x}\right\rangle+2 v\left\langle\sigma_{x} \sigma_{x-e_{1}}\right\rangle \geqq 2 m\left\langle\sigma_{x}\right\rangle+2 v\left\langle\sigma_{x}\right\rangle^{2}
$$


by the Schwarz inequality (3.56). Replacing the inequality by an equation, one gets the upper bound for $\left\langle\sigma_{x}\right\rangle$ by the mean-field solution. The lower bound is shown by expressing the right side of (4.10),

$$
\sum_{y:|y-x|=1}\left\langle\sigma_{x} \sigma_{y}\right\rangle=\sum_{|\xi|=1} T(\xi)
$$

in terms of the Fourier transform $\hat{T}$ and inserting the form (3.111) of the latter which was derived from the infrared bound,

$$
1-2 m s=2 \int_{\mathscr{\mathscr { O }} v} d^{v} k \hat{T}(k) C(k)=2 C(0) s^{2}+2 \int_{\mathscr{O}_{v}} d^{v} k \hat{g}(k) C(k)
$$

with $s=\left\langle\sigma_{x}\right\rangle \in[0,1]$ and $C(k)$ given by (4.1). Since $C(k+\hat{\pi})=-C(k)$, $\mathscr{B}_{v}^{+}+\hat{\pi}=\mathscr{B}_{v} \backslash \mathscr{B}_{v}^{+}$up to a set of measure zero, and

$$
\int_{\mathscr{H}_{v}} d^{v} k \hat{g}(k) C(k)=\int_{\mathscr{O}_{v}^{+}} d^{v} k C(k)(\hat{g}(k)-\hat{g}(k+\hat{\pi})) \leqq \frac{1}{N} S(v),
$$

where the last inequality follows from (3.109) which can be applied because $C>0$ on $\mathscr{B}_{v}^{+} . C(0)=v$, thus

$$
1-2 m s \leqq 2 v s^{2}+\frac{2}{N} S(v)
$$

which implies the lower bound by the solution of (4.9).

Corollary 4.4. Let $v \geqq 3$.

(1) Chiral symmetry is broken for all $N, v$ for which $2 S(v) / N<1$, the chiral order parameter $X=\liminf _{m \rightarrow 0}\langle\bar{\psi} \psi\rangle$ satisfies

$$
\frac{1}{\sqrt{2 v}} \geqq \frac{X}{2 N} \geqq \frac{1}{\sqrt{2 v}}\left(1-\frac{2}{N} S(v)\right)^{1 / 2} .
$$

In the case $N=1$, which is strongly coupled (compact or noncompact) QED, $2 S(v) / N<1$ holds for all $v \geqq 4$, so chiral symmetry is broken for all $v \geqq 4$. If $N \geqq 2$ the breaking of the symmetry holds for all $v \geqq 3$.

(2) Mean field theory, which neglects all fluctuations, is an upper bound for the expectation value of $\langle\bar{\psi} \psi\rangle$. The lower bound is of precisely the same form, except that in it the effect of the fluctuations has been included and bounded by $2 S(v) / N$. Therefore, as $N \rightarrow \infty$ or $v \rightarrow \infty$, the fluctuations die out, the lower bound $s_{2}$ approaches the upper one $s_{1}$ and the mean-field solution indeed becomes exact in either of these limits. An explicit lower bound for $X$ for $v \geqq v_{0}$ $\left(v_{0}\right.$ specified in the appendix), is

$$
X \geqq \frac{2 N}{\sqrt{2 v}}\left(1-\frac{2 c\left(v_{0}\right)}{N v^{1 / 4}}\right)^{1 / 2} .
$$

(3) The upper bound for $\langle\bar{\psi} \psi\rangle$ by the mean field solution decreases with increasing mass $m$, in particular

$$
\langle\bar{\psi} \psi\rangle \leqq N \min \left\{\sqrt{\frac{2}{v}}, \frac{1}{m}\right\},
$$

so the actual influence of $m$ on the order parameter is not similar to that of an external field in an Ising system, contrary to what the way in which $m$ appears in the action might suggest. 
(4) The chiral Ward identity (3.43) and $X \neq 0$ imply that the clustering rate $\kappa(m)$ satisfies.

as $m \rightarrow 0$.

$$
\kappa(m) \leqq \text { const } m^{1 / \nu}
$$

Proof. Clear from the preceding Theorem and the properties of $S(v)$ listed in Proposition 4.2.

\section{$U(N)$ Model: The Two-Point Function Declines to Decay}

The treatment of the $U(N)$-model for $N \geqq 2$ faces some difficulties due to the more complicated interaction term $W$. Whereas the infrared bound can be shown to carry over unchanged, the lower bound does not because contributions of the form $\left\langle\left(\sigma_{x} \sigma_{x+e_{\mu}}\right)^{k}\right\rangle$ are present in the SDE. It is possible to bound them in terms of $\left\langle\sigma_{x} \sigma_{x+e_{\mu}}\right\rangle$, but the constants get worse when this is done. Last but not least the proof that clustering holds for nonzero mass has not been extended to the $U(N)$-models for $N \geqq 2$, therefore only existence of long-range order can be shown.

The most general result about LRO in complex spin systems which is obtained here is Theorem 4.8. Let $N \in \mathbb{N}$, and $\langle\cdot\rangle_{A}$ denote the expectation value of the $U(N)$ model on $\Lambda$ as defined in (3) of Definition 3.3, if nothing else is stated. Without of loss of generality, $m \geqq 0$.

Remark 4.5. The $U(N)$-model fulfills the requirements of Theorem 3.21, so the infrared bound $(3.74,3.75)$ holds.

Proof. The only thing to be proven is that the coefficients in the expansion of the exponential of $V(t)=W(t)-t$ are nonnegative. Since $g_{4} \geqq 0$ and $g_{4}$ enters in $B$ as given by (3.8), it suffices to give a proof for $g_{4}=0$. It is convenient (see (3.8)) to consider the expansion with respect to $u=N^{2} t$. The problem is then to show that in the expansion

$$
e^{-u / N} \sum_{k=0}^{N} \frac{(N-k) !}{N ! k !} u^{k}=\sum_{k \geqq 0} b_{k} u^{k}
$$

$b_{k} \geqq 0$ for $k \in\{1, \ldots, N\}$. By construction, $b_{1}=0$, and

$$
b_{m}=\sum_{k=0}^{m} \frac{(N-k) !}{N ! k !} \frac{(-1)^{m-k}}{(m-k) ! N^{m-k}} .
$$

Using the Euler representation for the factorial in the numerator,

$$
b_{m}=\frac{1}{m ! N !} \int_{0}^{\infty} d t e^{-t}\left(1-\frac{t}{N}\right)^{m} t^{N-m}
$$

which shows already that $b_{m} \geqq 0$ for even $m \leqq N$. Introducing $c_{m}=m ! N ! b_{m}$ and applying integration by parts (writing $t^{N-m}$ as a derivative),

with

$$
(N-(m-1)) c_{m}=\left(1+\frac{m}{N}\right) c_{m-1}-\frac{1}{N} d_{m}
$$

$$
d_{m}=\int_{0}^{\infty} d t e^{-t}\left(1-\frac{t}{N}\right)^{m-1} t^{N+2-m}
$$


A second integration by parts, taking $e^{-t}=-\left(e^{-t}\right)^{\prime}$, yields

$$
\frac{1}{N} d_{m}=\left(1-\frac{m-2}{N}\right) c_{m-1}-\frac{m-1}{N^{2}} c_{m-2}
$$

and thus the recursion relation

$$
(N-m+1) c_{m}=\frac{2}{N}(m-1) c_{m-1}+\frac{m-1}{N^{2}} c_{m-2} .
$$

Since $c_{0}=N$ ! and $c_{1}=0$, this implies $c_{m} \geqq 0$ for all $m \in\{0, \ldots, N\}$.

Remark 4.6. Let $N \leqq 5$. Then $w_{k} \geqq 0$ for all $k \leqq N$ holds in the $U(N)$-model, because

$$
\begin{aligned}
& w_{1}=1 \\
& w_{2}=\frac{1}{2} \frac{N}{N-1}, \\
& w_{3}=\frac{2 N^{2}}{3(N-1)(N-2)}, \\
& w_{4}=\frac{(5 N-6) N^{3}}{4(N-1)^{2}(N-2)(N-3)}, \\
& w_{5}=\frac{2 N^{4}(5 N-12)}{5(N-1)^{2}(N-2)(N-3)(N-4)} .
\end{aligned}
$$

Lemma 4.7. Let $w_{k} \geqq 0$ for all $k \in\{0, \ldots, N\}, w_{1}=1$ and $m \geqq 0$. Then

$$
\left\langle\left(\sigma_{x} \sigma_{x+e_{1}}\right)^{n}\right\rangle \leqq \alpha_{n}\left\langle\sigma_{x} \sigma_{x+e_{1}}\right\rangle,
$$

where

$$
\alpha_{n}=\frac{\prod_{p=1}^{n-1}\left(1-\frac{p}{N}\right)}{1+\sum_{k=2}^{n-1} k w_{k} \sum_{l=1}^{k-1}\left(1-\frac{n-l}{N}\right)} .
$$

Proof. Abbreviate

$$
S_{n}=\left\langle\left(\sigma_{x} \sigma_{x+e_{1}}\right)^{n}\right\rangle
$$

and apply the SD equation (3.46) to $\sigma^{L}=\left(\sigma_{x} \sigma_{x+e_{1}}\right)^{n}$, to get

$$
\begin{aligned}
\left(1-\frac{n}{N}\right) S_{n} & =2 m\left\langle\sigma_{x}\left(\sigma_{x} \sigma_{x+e_{1}}\right)^{n}\right\rangle \\
& +\sum_{\mu=1}^{v}\left\langle\left(\sigma_{x} \sigma_{x+e_{1}}\right)^{n}\left(\sigma_{x} \sigma_{x+e_{\mu}} W^{\prime}\left(\sigma_{x} \sigma_{x+e_{\mu}}\right)+\sigma_{x} \sigma_{x-e_{\mu}} W^{\prime}\left(\sigma_{x} \sigma_{x-e_{\mu}}\right)\right)\right\rangle,
\end{aligned}
$$

stated otherwise,

$$
S_{n+1}+\sum_{k=2}^{N-n} k w_{k} S_{n+k}=\left(1-\frac{n}{N}\right) S_{n}-R_{n}-T_{n},
$$

where

$$
R_{n}=2 m\left\langle\sigma_{x}\left(\sigma_{x} \sigma_{x+e_{1}}\right)^{n}\right\rangle \geqq 0
$$


and

$$
\begin{aligned}
T_{n}= & \left\langle\left(\sigma_{x} \sigma_{x+e_{1}}\right)^{n} \sigma_{x} \sigma_{x-e_{1}}\right\rangle \\
& +\left\langle\left(\sigma_{x} \sigma_{x+e_{1}}\right)^{n}\left(\sigma_{x} \sigma_{x+e_{\mu}} W^{\prime}\left(\sigma_{x} \sigma_{x+e_{\mu}}\right)+\sigma_{x} \sigma_{x-e_{\mu}} W^{\prime}\left(\sigma_{x} \sigma_{x-e_{\mu}}\right)\right)\right\rangle \geqq 0
\end{aligned}
$$

are nonnegative, the reason being again that the same is true for all $w_{k}$. Dropping $R_{n}$ and $T_{n}$, and calling $n+1=a$, this becomes the inequality

$$
S_{a} \leqq\left(1-\frac{a-1}{N}\right) S_{a-1}-\sum_{b=2}^{N+1-a} b w_{b} S_{a-1+b}
$$

which holds for all $a \in\{1, \ldots, N\}$ (empty sums are to be interpreted as zero). Let $n \in\{1, \ldots, N\}$. We will prove by induction that for all $k \in\{1, \ldots, n-1\}$

$$
S_{n}\left(1+\sum_{r=2}^{k} r w_{r} \prod_{s=1}^{r-1}\left(1-\frac{n-s}{N}\right)\right) \leqq S_{n-k} \prod_{l=1}^{k}\left(1-\frac{n-l}{N}\right) .
$$

For $k=1$, the claim $S_{n} \leqq S_{n-1}(1-(n-1) / N)$ follows directly from (4.35). Assume now that (4.36) has been proven up to $k \in\{1, \ldots, n-2\}$. From (4.35) with $a=n-k$,

$$
\begin{aligned}
S_{n-k} & \leqq\left(1-\frac{n-k-1}{N}\right) S_{n-k-1}-\sum_{u=2}^{N+1-n+k} u w_{u} S_{n-k-1+u} \\
& \leqq\left(1-\frac{n-k-1}{N}\right) S_{n-(k+1)}-(k+1) w_{k+1} S_{n} .
\end{aligned}
$$

The induction step is completed by inserting this bound for $S_{n-k}$ on the right side of (4.36) and transferring the term proportional to $S_{n}$ to the left side. The case $k=n-1$ is the assertion of the Lemma.

Theorem 4.8. Let $N \in \mathbb{N}$ and the interaction $W$ of the complex spin system have coefficients $w_{k} \geqq 0$ for all $k \in\{0, \ldots, N\}$ and $w_{1}=1$. Then there is $a v_{0} \geqq 3$ so that long-range order holds at $m=0$ for all $v \geqq v_{0}$.

Proof. Due to the nonnegativity of the $w_{k}$, Theorem 3.18 applies and guarantees the existence of thermodynamic limits as well as boundedness of the two-point function. As in the proof of Theorem 4.3, the starting point is the infinite-volume SD equation for $L=0$ which now reads

$$
1=2 m\left\langle\sigma_{x}\right\rangle+\sum_{k=1}^{N} k w_{k} \sum_{|y-x|=1}\left\langle\left(\sigma_{x} \sigma_{y}\right)^{k}\right\rangle \leqq 2 m\left\langle\sigma_{x}\right\rangle+K(N) \sum_{|y-x|=1}\left\langle\sigma_{x} \sigma_{y}\right\rangle,
$$

the inequality comes from application of Lemma 4.7 , with

$$
K(N)=\sum_{k=1}^{N} k w_{k} \alpha_{k}
$$

$\alpha_{1}=1$, and $\alpha_{k}, k \geqq 2$ given by (4.29). Theorem 3.23 applies, so again

$$
\begin{aligned}
\sum_{|y-x|=1}\left\langle\sigma_{x} \sigma_{y}\right\rangle & =2 \int \frac{d^{v} k}{(2 \pi)^{v}} \hat{T}(k) C(k)=2 v\left(c_{0}+c_{\hat{\pi}}\right)+2 \int \frac{d^{v} k}{(2 \pi)^{v}} \hat{g}(k) C(k) \\
& \leqq 2 v\left(c_{0}+c_{\hat{\pi}}\right)+\frac{2 S(v)}{N} .
\end{aligned}
$$


At $m=0, c_{0}=c_{\hat{\pi}}$ because of chiral symmetry, (3.106), so

$$
1 \leqq K(N)\left(4 v c_{0}+\frac{2 S(v)}{N}\right)
$$

and

$$
c_{0} \geqq \min \left\{0, \frac{1}{4 v}\left(\frac{1}{K(N)}-\frac{2 S(v)}{N}\right)\right\}>0
$$

for $v \geqq v_{0}$, where $2 S(v) K(N) / N<1$. $v_{0}$ exists because $S(v) \rightarrow 0$ as $v \rightarrow \infty$ according to Proposition 4.2.

Corollary 4.9. For $N \leqq 4$ and $v \geqq 4$, the $U(N)$-model has chiral LRO at $m=0$, for $N=5$ the same is true for $v \geqq 5$.

Proof. $K(N)$ is calculated using Remark 4.6 and Lemma 4.7; $K(1)=1$, $K(2)=2, K(3)=\frac{10}{3}, K(4)=5+\frac{8}{15}, K(5)<7.4$. That $c_{0}>0$ in the cases mentioned follows from the numbers given for $S(v)$.

In [36] it was stated that the proof works also for $N=5$ and $v=4$, i.e. that also $2 S(4) \frac{K(5)}{5}<1$. This statement is incorrect due to a calculational error in the determination of $K(5)$ made in [36].

Remark 4.10. (1)

$$
\limsup _{x \in \mathbb{Z}^{\nu}} \frac{1}{(2 N)^{2}}\langle\bar{\psi} \psi(0) \bar{\psi} \psi(x)\rangle=\lim _{|x| \rightarrow \infty, \varepsilon(x)=-1} \frac{1}{(2 N)^{2}}\langle\bar{\psi} \psi(0) \bar{\psi} \psi(x)\rangle=2 c_{0} .
$$

(2) Assuming that $w_{k} \geqq 0$ if $k \leqq N$ is true for all $N \in \mathbb{N}$, chiral LRO can be proven in the $U(N)$-model for $v \geqq v_{0}(N)$. From the $1 / N$-expansion, one would expect the fluctuations to decrease with increasing $N$ which is very likely to happen; unfortunately, inequality (4.29) is still too crude to allow for a proof of this.

(3) Assuming that clustering holds for $m>0$ also in the $U(N)$-model one can again get a lower bound for the chiral order parameter

$$
\left\langle\sigma_{x}\right\rangle \geqq s_{3},
$$

where

$$
2 v K(N) s_{3}^{2}+2 m s_{3}-1+\frac{2 K(N)}{N} S(v)=0
$$

$[K(N)$ as in the proof of Theorem 4.8] which means that chiral symmetry is broken in the sense $X \neq 0$ whenever chiral LRO holds.

(4) It is generally expected in the spirit of phase decompositions that symmetry breaking and existence of LRO are equivalent. For the NJL model, $W(t)=t, K(N)=1$ and by Corollaries 4.4 and 4.9 we have a proof that chiral LRO holds if and only if the chiral condensate has a nonvanishing limit as the mass goes to zero. 


\section{Summary}

The material presented here consists of some already known and some new rigorous results about the phase structure of lattice QED, gauged NambuJona-Lasinio models and models with gauge group $U(N)$. The new results concern the strongly coupled theory with staggered fermions, i.e. the theory in the limit of infinite bare coupling, and contain

(1) the proof of clustering of truncated expectation values for QED and the NJL-systems at nonzero mass (Theorem 3.11),

(2) the proof that chiral symmetry is broken in QED and the NJL models if the dimension is at least four (Theorem 4.3, Corollary 4.4),

(3) a lower bound for the chiral order parameter in thse systems which implies that mean field theory becomes exact for dimension to infinity at least for this observable (Theorem 4.3, Corollary 4.4),

(4) the proof of existence of chiral long-range order in the models with gauge group $U(N)$ for $N \leqq 4$ if the dimension is at least four (Corollary 4.9).

That we have to require $N \leqq 4$ is a purely technical limitation which might be overcome if the constants can be improved. The case of $S U(N)$ is technically more complicated; the set of $S U(N)$-invariants is larger than that of $U(N)$ invariants, which gives rise to other than nearest-neighbour interactions in the effective fermionic theory (these objects are aptly called baryon loops in [30]). However, it is generally believed that these additional terms do not qualitatively change the behaviour and assuming this our results give support for chiral symmetry breaking in QCD with massless quarks.

For $N=1$, the results establish the existence of a phase of lattice QED in which chiral symmetry is broken spontaneously and thus provide a proof of some of the assumptions on which the present discussion about a possible nontrivial continuum limit of lattice QED is based. The known results about monomer-dimer systems and the techniques used in the proofs, namely the infrared bound and integration by parts formulas, may suffice to extend analyticity of the expectation values and clustering at nonzero mass and chiral symmetry breaking in the limit as the mass vanishes to large but finite gauge coupling in compact QED. Among the many open mathematical problems which further work about lattice QED should attack the most important question seems to be whether a chirally symmetric phase at weak coupling exists.

\section{Appendix}

Let $v>2$. The purpose of the following is to study the function

$$
S(v)=\int_{\mathscr{S}_{v}} \frac{d^{v} k}{(2 \pi)^{v}} \frac{C(k)}{D(k)}=\int_{\mathscr{B}_{v}} \frac{d^{v} k}{(2 \pi)^{v}} \frac{C(k)}{D(k)} \Theta(C(k))
$$

[see $(4.1,4.2,4.3), \Theta(x)=1$ if $x>0$ and zero otherwise], especially its dependence on the dimension $v$, and to prove Proposition 4.2. To do so, it is convenient to rewrite the integral such that the $v$-dependence is only para- 
metric, i.e. that the integration measure does not change with $v$. Noting that $C(k)=v-D(k)$, it is possible to split

$$
\begin{aligned}
S(v) & =v R_{+}(v)-\frac{1}{2}, \\
R_{+}(v) & =R(v)-R_{-}(v), \\
R(v) & =\int_{\mathscr{O}_{v}} \frac{d^{v} k}{(2 \pi)^{v}} \frac{1}{D(k)}, \\
R_{-}(v) & =\int_{\mathscr{O}_{v}} \frac{d^{v} k}{(2 \pi)^{v}} \frac{\Theta(-C(k))}{D(k)}=\int_{\mathscr{O}_{v}} \frac{d^{v} k}{(2 \pi)^{v}} \frac{\Theta(C(k))}{v+C(k)} .
\end{aligned}
$$

The integrand in $R_{-}(v)$ is a bounded function if $v>0$, therefore the integral exists for all $v>0$. The singularity at $k=0$ is in the integrand of the expression for $R(v)$.

Remark A.1. Since $0 \leqq C(k) \leqq v$ throughout the support of the integrand in (A.5), and $\int \Theta(C(k)) \bar{d} k /(2 \pi)^{v}=\frac{1}{2}$,

$$
\frac{1}{4 v} \leqq R_{-}(v) \leqq \frac{1}{2 v} .
$$

Proposition A.2. Let $v \in \mathbb{N}$.

(1) Let $v>2, r(x)=I_{0}(x) e^{-x}$, where $I_{0}$ is the modified Bessel function of zero ${ }^{\text {th }}$ order. Then

$$
R(v)=\int_{0}^{\infty} r(x)^{v} d x
$$

(2) Let $J_{0}$ be the zero ${ }^{\text {th }}$ Bessel function and for $\alpha>0$,

$$
f(\alpha)=\int_{0}^{\alpha} \frac{\cos x}{x+\alpha} d x
$$

then

$$
R_{-}(v)=\frac{1}{\pi} \int_{0}^{\infty} d \tau J_{0}(\tau)^{v} f(v \tau)
$$

Remark A.3. The integral (A.7) defines a continuation of $R$ to real values of $v>2$, so $v$ can be regarded as a real variable. Whenever derivatives of $R$ with respect to $v$ occur in the following they are meant to act on the function defined by (A.7).

Proof of Proposition A.2. Let $v \in \mathbb{N}, v>2$. Then the integral (A.4) is absolutely convergent, and therefore

$$
R(\varepsilon, v)=\int_{\mathscr{B} v} \frac{d^{v} k}{(2 \pi)^{v}} \frac{1}{\varepsilon^{2}+D(k)}
$$

is defined for $\varepsilon \in \mathbb{R}$ and continuous at zero:

$$
\lim _{\varepsilon \rightarrow 0} R(\varepsilon, v)=R(0, v)=R(v) .
$$


Let $\varepsilon \neq 0$, then $\varepsilon^{2}+D(k)>0$ and

$$
\frac{1}{\varepsilon^{2}+D(k)}=\int_{0}^{\infty} d t e^{-t\left(\varepsilon^{2}+D(k)\right)}
$$

the integral converges uniformly in $k$. Inserting this into (A.10), one can therefore integrate over $k$ first, and now this integral is a product of onedimensional ones:

$$
R(\varepsilon, v)=\int_{0}^{\infty} e^{-\varepsilon^{2} t} \int_{\mathscr{B}_{v}} \frac{d^{v} k}{(2 \pi)^{v}} e^{-t\left(v-\sum_{\mu=1}^{v} \cos k_{\mu}\right)}=\int_{0}^{\infty} e^{-\varepsilon^{2} t} e^{-v t} I_{0}(t)^{\nu}
$$

due to the standard integral representation [48] of $I_{0}$. The asymptotic behaviour of $I_{0}(x)$ as $x \rightarrow \infty$ is

$$
I_{0}(x) \sim \frac{1}{\sqrt{2 \pi x}} e^{x}\left(1+O\left(\frac{1}{x}\right)\right)
$$

therefore the integrand behaves as $t^{-\frac{v}{2}}$ for large $t$, and the integral converges absolutely for $v>2$, independently of $\varepsilon$. The limit $\varepsilon \rightarrow 0$ can therefore be taken under the integral, which yields (A.7).

The integral (A.5) for $R_{-}$can be rewritten as

$$
R_{-}(v)=\int_{0}^{v} \frac{d \xi}{v+\xi} \int_{\mathscr{B}_{v}} \delta(\xi-C(k)) \frac{d^{v} k}{(2 \pi)^{v}} .
$$

[Strictly speaking, the function $\Theta$ should be mollified to some $\tilde{\Theta} \in \mathscr{S}\left(\mathscr{B}_{v}\right)$ to be able to apply $\delta$ as a distribution. This is completely analogous to the procedure in the preceding proof.] To evaluate the integral over hypersurfaces of constant $C(k), \delta$ is replaced by

and

$$
\delta_{\varepsilon}(u)=\frac{1}{\pi} \int_{0}^{\infty} d t e^{-\varepsilon t} \cos (u \tau)
$$

$$
R_{-}(v)=\lim _{\varepsilon \rightarrow 0} R_{-}(\varepsilon, v) .
$$

Using the standard integral representation for $J_{0}[48]$,

$$
R_{-}(\varepsilon, v)=\frac{1}{\pi} \int_{0}^{\infty} d \tau e^{-\varepsilon \tau} J_{0}(\tau)^{\nu} f(\nu \tau)
$$

with $f$ given by (A.8). The limit $\varepsilon \rightarrow 0$ yields (A.9).

We will use the following elementary properties of the Bessel functions:

and for $x \geqq 0$,

$$
x^{2} I_{0}^{\prime \prime}(x)+x I_{0}^{\prime}(x)-x^{2} I_{0}(x)=0,
$$

and

$$
I_{0}^{(n)}(x) \geqq 0
$$

$$
\begin{aligned}
I_{0}^{\prime} & =I_{1}<I_{0}, \\
I_{1} & \leqq \frac{x}{2} I_{0}, \\
\frac{1}{2} I_{0} & \leqq I_{0}^{\prime \prime} \leqq I_{0} .
\end{aligned}
$$


Lemma A.4. (1) $r(x)=I_{0}(x) e^{-x}$ satisfies

$$
0 \leqq r(x) \leqq \frac{1}{\sqrt{1+2 x}}
$$

for all $x \geqq 0$. The upper bound becomes exact as $x \rightarrow 0$.

(2)

$$
R(v) \leqq \frac{1}{v-2}
$$

Proof. By the integral representation for $I_{0}$,

$$
r(x)=\frac{1}{2 \pi} \int_{-\pi}^{\pi} e^{x(\cos t-1)} d t
$$

so

$$
r^{\prime \prime}(x)=\frac{1}{2 \pi} \int_{-\pi}^{\pi} e^{x(\cos t-1)}(\cos t-1)^{2} d t>0
$$

for all $x \in \mathbb{R}$. The differential equation (A.19) for the Bessel function $I_{0}$ implies

$$
x r^{\prime \prime}(x)+(1+2 x) r^{\prime}(x)+r(x)=0 .
$$

For $x \geqq 0, x r^{\prime \prime} \geqq 0$, so

$$
(1+2 x) r^{\prime}(x)+r(x) \leqq 0,
$$

which, upon integration, gives (A.22). (A.7) then gives (A.23).

Remark A.5. By (A.2, A.3, A.6) and, for the second inequality, (A.23),

$$
S(v) \leqq v R(v)-\frac{3}{4} \leqq \frac{v}{v-2}-\frac{3}{4} .
$$

Proposition A.6. Let $v \in \mathbb{R}, v \geqq 3$. Then $v R(v)$ is a decreasing function of $v$.

Proof. Let $F(v)=v R(v)$. From (A.7),

$$
v R^{\prime}(v)=\int_{0}^{\infty} d x v e^{-v x} I_{0}(x)^{v}\left(\log I_{0}(x)-x\right) .
$$

Using integration by parts with $v e^{-v x}=-\frac{d}{d x} e^{-v x}$,

$$
v R^{\prime}(v)=\int_{0}^{\infty} d x e^{-v x} I_{0}(x)^{v-1} I_{1}(x)\left(v\left(\log I_{0}(x)-x\right)+1\right)-R(v) .
$$

Again integrating by parts in the summand multiplied by $v$,

with

$$
F^{\prime}(v)=\int_{0}^{\infty} d x e^{-v x} I_{0}(x)^{v-2} \varphi(x)
$$

$$
\varphi(x)=\left(\log I_{0}(x)-x\right)\left((v-1) I_{1}(x)^{2}+I_{0}(x) I_{1}(x)\right)+I_{1}(x)^{2} .
$$

By (A.22),

$$
\log I_{0}(x)-x \leqq-\frac{1}{2} \log (1+2 x) \leqq 0
$$


for all $x \geqq 0$, so the only positive contribution to $\varphi$ comes from the term $I_{1}^{2}$,

$$
\varphi(x) \leqq I_{1}(x)^{2}\left((v-1)\left(\log I_{0}(x)-x\right)+1\right)
$$

which is negative for $x \geqq x_{0}=\frac{1}{2}\left(e^{2 /(v-1)}-1\right)$. For $v \geqq 3, x_{0}<1$. For $x \leqq 1$, one can use the bounds (A.21) to get

$$
\begin{aligned}
\varphi(x) & \leqq I_{0}(x)^{2}\left(\frac{x^{2}}{4}-\left(x-\log I_{0}(x)\right)\right)-\frac{I_{1}(x)^{2}}{I_{0}(x)^{2}}\left(x-\log I_{0}(x)\right) \\
& \leqq \frac{1}{4}\left(x^{2}-\log (1+2 x)\right) \leqq 0 .
\end{aligned}
$$

So, for $v \geqq 3, \varphi(x) \leqq 0$ for all $x \geqq 0$, and the derivative of $F$ with respect to $v$ is negative.

Lemma A.7. The upper bound in (A.6) becomes exact as $v \rightarrow \infty$,

$$
R_{-}(v)=\frac{1}{2 v}(1-u(v))
$$

$u(v) \geqq 0, u(v) \rightarrow 0$ as $v \rightarrow \infty$. For $v \geqq v_{0}$,

$$
u(v) \leqq \frac{c\left(v_{0}\right)}{v^{1 / 4}} .
$$

Proof. Let $\alpha \in \mathbb{R}, 0<\alpha<\frac{1}{3}$, and split

$$
R_{-}(v)=\frac{1}{\pi v} \int_{0}^{\infty} d t J_{\mathrm{o}}\left(\frac{t}{v}\right)^{v} f(t)=\frac{1}{\pi v}\left(\varrho_{1}+\varrho_{2}+\varrho_{3}\right),
$$

where

$$
\begin{aligned}
& \varrho_{1}=\int_{0}^{v^{\alpha}} d t J_{0}\left(\frac{t}{v}\right)^{v} f(t), \\
& \varrho_{2}=\int_{v^{\alpha}}^{v} d t J_{0}\left(\frac{t}{v}\right)^{v} f(t), \\
& \varrho_{3}=\int_{v}^{\infty} d t J_{0}\left(\frac{t}{v}\right)^{v} f(t)=v \int_{1}^{\infty} d \tau J_{0}(\tau) f(v \tau) .
\end{aligned}
$$

The function $f$ has the following properties:

$$
|f(t)| \leqq \int_{0}^{t} \frac{d x}{t+x}=\log 2 \leqq 1
$$

for all $t>0$, and (by double integration by parts)

$$
f(t)=\frac{\sin t}{2 t}+\frac{1}{t^{2}} \varphi(t)
$$

with $|\varphi(t)| \leqq 3$ for all $t$. Let $v>2$, then $\int J_{0}(\tau) d \tau$ converges absolutely because $J_{0}(x)=O\left(x^{-1 / 2}\right)$ for large $x$. Since $\left|J_{0}(x)\right| \leqq J_{0}(1) \leqq e^{-1 / 4}$ for all $x \geqq 1$ and $f$ is bounded,

$$
\left|\varrho_{3}\right| \leqq \text { const } e^{-v / 4}
$$


for large $v$. From (A.41),

$$
\varrho_{2}=\int_{v^{\alpha}}^{v} d t J_{0}\left(\frac{t}{v}\right)^{v}\left(\frac{\sin t}{2 t}+\frac{\varphi(t)}{t^{2}}\right) .
$$

$0 \leqq J_{0}(x) \leqq 1$ for all $x \in[0,1]$, and $\varphi$ is bounded, so

$$
\left|\int_{\nu^{\alpha}}^{v} J_{0}\left(\frac{t}{v}\right)^{v} \frac{\varphi(t)}{t^{2}}\right| \leqq 3 \int_{v^{\alpha}}^{v} t^{-2} d t \leqq 3 v^{-\alpha} .
$$

The remaining integral can be decomposed according to the periods of the sine,

$$
\int_{v^{\alpha}}^{v} d t J_{0}\left(\frac{t}{v}\right)^{v} \frac{\sin t}{2 t}=\int_{v^{\alpha}}^{m \pi} d t J_{0}\left(\frac{t}{v}\right)^{v} \frac{\sin t}{2 t}+(-1)^{m} \sum_{n \geqq 0}(-1)^{n} a_{n}(v),
$$

where

$$
\frac{v^{\alpha}}{\pi} \leqq m=\min \left\{l \in \mathbb{Z} \mid l \pi \geqq v^{\alpha}\right\} \leqq 1+\frac{v^{\alpha}}{\pi}
$$

and

$$
a_{n}(v)=\int_{(m+n) \pi}^{(m+n+1) \pi} d t J_{0}\left(\frac{t}{v}\right)^{v} \frac{|\sin t|}{2 t} \Theta(v-t) .
$$

Since $J_{0}$ is positive in the integration interval, $a_{n}(v) \geqq 0$ for all $n \geqq 0$, and

$$
a_{n+1}(v)<a_{n}(v)
$$

for all $n \geqq 0$ for which $a_{n}>0$ because $J_{0}$ is a strictly decreasing function on $(0,1]$. By Leibniz's criterion this implies

$$
0 \leqq \sum_{n \geqq 0}(-1)^{n} a_{n}(v) \leqq a_{0}(v),
$$

and some simple estimates yield

$$
\left|\varrho_{2}\right| \leqq 3 v^{-\alpha}+\frac{\pi}{2 m} \leqq v^{-\alpha}\left(3+\frac{\pi^{2}}{2}\right)
$$

Again by the Leibniz criterion, now applied to the expansion of $J_{0}$ around zero, for all $x \leqq 2$,

$$
\left(1-\frac{x^{2}}{4}\right) \leqq J_{0}(x) \leqq\left(1-\frac{x^{2}}{8}\right)^{2}
$$

therefore

$$
0 \leqq 1-J_{0}(x)^{v} \leqq 1-\left(1-\frac{x^{2}}{4}\right)^{v}=v \int_{0}^{x^{2}}(1-\xi)^{v-1} d \xi \leqq v \frac{x^{2}}{4},
$$

and

$$
\left|\int_{0}^{v^{\alpha}} d t\left(1-J_{0}\left(\frac{t}{v}\right)^{v}\right) f(t)\right| \leqq v \int_{0}^{v^{\alpha}} \frac{t^{2}}{4 v^{2}} d t=\frac{1}{12} v^{3 \alpha-1}
$$

Therefore

$$
\left|\varrho_{1}-\int_{0}^{v^{\alpha}} f(t) d t\right| \leqq \frac{1}{12} v^{3 \alpha-1},
$$


and the remaining task is to calculate the integral over $f$ alone. Interchanging the order of integrations and performing an integration by parts we get

$$
\int_{0}^{v^{\alpha}} f(t) d t=\operatorname{Si}(0)-\operatorname{Si}\left(v^{\alpha}\right)+\left(\sin \left(v^{\alpha}\right)-\cos \left(v^{\alpha}\right)\right)\left(\operatorname{Si}\left(v^{\alpha}\right)-\operatorname{Si}\left(2 v^{\alpha}\right)\right) .
$$

The function

$$
S i(x)=\int_{x}^{\infty} \frac{\sin \xi}{\xi} d \xi
$$

satisfies

$$
|S i(x)| \leqq \frac{2}{x-\pi}
$$

for $x>\pi$ and

$$
\operatorname{Si}(0)=\frac{\pi}{2} \text {. }
$$

For $\alpha=1 / 4$, (A.42, A.50, A.53, A.55, A.58) imply (A.36) and (A.37), with some constant $c\left(v_{0}\right)$.

Proof of Proposition 4.2, (1)-(3). (1) is clear, (2) follows from Remark A.5 and Proposition A.6. (3) From (A.2, A.3, A.23) and (A.36),

$$
0 \leqq S(v) \leqq \frac{v}{v-2}-v R_{-}(v)-\frac{1}{2}=\frac{v}{v-2}-1+\frac{1}{2} u(v) \rightarrow 0
$$

as $v \rightarrow \infty$.

The numbers in (4) of Proposition 4.2 have been obtained doing the integrals (A.7), (A.9) numerically, which is a straightforward exercise. However, since the concrete values of $v$ and $N$ for which chiral symmetry breaking holds depend on these constants some words about how they were obtained may be in place.

Concerning the calculation of $R(v)$, since the integrand in (A.7) is a monotonically decreasing function which is convex by (A.25), one can always majorize it by a piecewise linear map in a finite interval and by an inverse power for large $|x|$, and thus get arbitrarily accurate upper bounds for $R(v)$, which are $R(3) \leqq 0.5062, R(4) \leqq 0.3100, R(5) \leqq 0.2313, R(6) \leqq$ $0.1862, \quad R(7) \leqq 0.1564, \quad R(8) \leqq 0.1349, \quad R(9) \leqq 0.1187, \quad R(10) \leqq 0.1061$ (the value of $R$ is known exactly for $v=3[18], R(3)=0.50546 \ldots)$. It may be noted that

$$
S(4) \leqq 4 R(4)-\frac{3}{4}<\frac{1}{2},
$$

so that already this bound suffices to prove symmetry breaking for QED, the NJL model and the $U(2)$-model for $v=4$ (and also all $v \geqq 4$, as will be seen below).

The integrand in (A.9) is a product of the oscillating function $f(v t)$ and $J_{0}(t)^{v}$ which also oscillates if $v$ is odd. The integral can safely be calculated by splitting the integration region into intervals between the zeros of $J_{0}$ and using asymptotic representations for large $t$. The actual analysis shows that because of the oscillations the contributions from the intervals between the zeros are almost negligible already after the third zero. Combining the results, one gets $S(3) \leqq 0.63, S(4) \leqq 0.34, S(5) \leqq 0.25, S(6) \leqq 0.20$. These num- 
bers suggest that actually $S(v)$ itself, not only the upper bound in terms of $R$, is a decreasing function of $v$.

Computer Assisted Proof of Proposition 4.2, (4). The numbers for $v=3,4,5$ have been give above. The inequality follows from the fact that the upper bound

$$
S(v) \leqq v R(v)-\frac{3}{4}
$$

is decreasing (see Proposition A.2) and the observation that already its value for $v=8,8 R(8)-3 / 4<S(4)$.

Acknowledgements. We are indebted to David Brydges for instructive discussions about the relation between compact and noncompact QED. M.S. is grateful to the Max-PlanckGesellschaft for its financial support.

\section{References}

1. Nambu, Y.: Phys. Rev. Lett. 4, 380 (1960)

2. Nambu, Y., Jona-Lasinio, G.: Phys. Rev. 122, 345 (1961)

3. Kogut, J. B.: Rev. Mod. Phys. 55, 775 (1983)

4. Thirring, W.: Ann. Phys. 3, 91 (1958)

5. Mitter, P. K., Weisz, P.H.: Phys. Rev. D 8, 4410 (1973)

6. Gross, D., Neveu, A.: Phys. Rev. D 10, 3235 (1974)

7. Mermin, N. D., Wagner, H.: Phys. Rev. Lett. 17, 1133 (1966)

8. Dobrushin, R. L., Shlosman, S. B.: Commun. Math. Phys. 42, 31 (1975)

9. Coleman, S.: Commun. Math. Phys. 31, 259 (1973); actually this was first proven by Maison, D., Reeh, H.: Commun. Math. Phys. 24, 67 (1971)

10. Miransky, V. A.: Nuovo Cimento 90 A, 149 (1985)

11. Kogut, J. B., Dagotto, E., Kocic, A.: Phys. Rev. Lett. 60, 772 (1988); 61, 2416 (1988); Nucl. Phys. B317, 253, 271 (1989)

12. Göckeler, M., Horsley, R., Laermann, E., Rakow, P., Schierholz, G., Sommer, R., Wiese, U.-J.: Nucl. Phys. B334, 527 (1990)

13. Hands, S., Kogut, J. B., Dagotto, E.: Nucl. Phys. B333, 551 (1990)

14. Göckeler, M., Horsley, R., Laermann, E., Wiese, U.-J., Rakow, P., Schierholz, G., Sommer, R.: Phys. Lett. 251 B, 567 (1990)

15. Lüscher, M.: Nucl. Phys. B341, 341 (1990)

16. Aoki, K. I.: Kyoto preprint RIFP-758 (1988)

17. Rakow, P.E. L.: Nucl. Phys. B356, 27 (1991)

18. Fröhlich, J., Simon, B., Spencer, T.: Commun. Math. Phys. 50, 79 (1976)

19. Fröhlich, J., Israel, R. B., Lieb, E. H., Simon, B.: Commun. Math. Phys. 62, 1 ( 9978$)$

20. Gruber, C., Kunz, H.: Commun. Math. Phys. 22, 133 (1971)

21. Heilmann, O. J., Lieb, E. H.: Commun. Math. Phys. 25, 190 (1972)

22. Fisher, M. E., Stephenson, J.: Phys. Rev. 132, 1411 (1963)

23. Kogut, J., Susskind, L.: Phys. Rev. D 11, 395 (1975)

Banks, T., Kogut, J., Susskind, L.: Phys. Rev. D 13, 1043 (1976)

Banks, T., Raby, S., Susskind, L., Kogut, J., Jones, D. R. T., Scharbach, P. N., Sinclair, D.: Phys. Rev. D 15, 1111 (1976)

Susskind, L.: Phys. Rev. D 16, 3031 (1977)

24. Sharatchandra, H., Thun, H., Weisz, P.: Nucl. Phys. B 192, 205 (1981)

25. Wilson, K.: Phys. Rev. D 10, 2455 (1974)

26. Berezin, F. A.: The method of second quantization. New York: Academic Press 1966

27. Bałaban, T., Brydges, D., Imbrie, J., Jaffe, A.: Ann. Phys. 158, 281 (1984)

28. Salmhofer, M.: PhD thesis, MPI-PAE-Th $80 / 90$

29. Lanford, O., Ruelle, D.: Commun. Math. Phys. 13, 194 (1969)

30. Rossi, P., Wolff, U.: Nucl. Phys. B 248, 105 (984)

Wolff, U.: Nucl. Phys. B 280, 680 (1987) 
31. Samuel, S.: J. Math. Phys. 21, 2695 (1980)

32. Bars, I.: J. Math. Phys. 21, 2678 (1980)

33. Brézin, E., Gross, D.: Phys. Lett. 97 B, 120 (1980)

34. Kluberg-Stern, H., Morel, A., Napoly, O., Petersson, B.: Nucl. Phys. B 190, 504 (1981)

Kluberg-Stern, H., Morel, A., Petersson, B.: Nucl. Phys. B215, 527 (1983)

35. Fisher, M. E.: Phys. Rev. 124, 1664 (1961)

36. Salmhofer, M., Seiler, E.: Lett. Math. Phys. 21, 13 (1991)

37. Mermin, N. D.: J. Math. Phys. 8, 1061 (1967)

38. Kasteleyn, P.W.: Physica, Grav. 27, 1209 (1961)

39. Temperley, H. N.V., Fisher, M. E.: Phil. Mag. Serie 86, 1664 (1961)

40. Kasteleyn, P.W.: J. Math. Phys. 4, 287 (1963)

41. McCoy, B., Wu, T.T.: The two-dimensional Ising model. Cambridge, MA: Harvard University Press 1973

42. Hartwig, R.: J. Math. Phys. 7, 286 (1966)

43. Penrose, O., Lebowitz, J. L.: Commun. Math. Phys. 39, 165 (1974)

44. Vladimirov, V.S.: Methods of the theory of functions of many complex variables. Cambridge, MA: M.I.T. Press 1966

45. Osterwalder, K., Seiler, E.: Ann. Phys. 110, 440 (1978)

46. Seiler, E.: Gauge theories as a problem of constructive quantum field theory and statistical mechanics. Lecture Notes in Physics, vol. 159. Berlin, Heidelberg, New York: Springer 1982

47. Reed, M., Simon, B.: Methods of modern mathematical physics, vol. 1. New York: Academic Press 1980

48. Erdélyi, A. (ed.): The Bateman manuscript project: higher transcendental functions, vol. 2. New York: McGraw-Hill 1953

Communicated by J. Fröhlich 